\title{
Cadmium im Grundwasser Nordwestdeutschlands - Herkunft, Mobilisierung und Bewertung nach EU-Wasserrahmenrichtlinie
}

\author{
Andreas Kubier $^{1}$ (D) $\cdot$ Dörte Budziak² $\cdot$ Dieter de Vries $^{3}$ Jörg Elbracht ${ }^{2} \cdot$ Kay Hamer $^{1} \cdot$ Thomas Pichler $^{1}$
}

Eingegangen: 24. Mai 2020 / Überarbeitet: 24. Februar 2021 / Angenommen: 19. Mai 2021 / Online publiziert: 18. Juni 2021

(c) Der/die Autor(en) 2021

\section{Zusammenfassung}

In Nordwestdeutschland zeigten neun Grundwasserkörper einen „schlechten“ chemischen Zustand nach Wasserrahmenrichtlinie aufgrund von Cadmiumkonzentrationen über 0,5 $\mu \mathrm{g} / \mathrm{l}$. Dünger- und Bodenanalysen, sowie Grundwasseranalysen des landesweiten Monitorings dienten der Quantifizierung der Cd-Belastung und Identifikation der Ursachen, wobei geogener Eintrag, Düngeremissionen und atmosphärische Deposition betrachtet wurden.

Bis 2016 sanken die Cadmium-Einträge auf einen mittleren Eintrag von $0,74 \mathrm{~g} /(\mathrm{ha} \cdot \mathrm{a})$ durch die Ausbringung von PhosphatDüngern und von $0,2 \mathrm{~g} /(\mathrm{ha} \cdot \mathrm{a})$ durch atmosphärische Deposition. In landwirtschaftlich genutzten Geestlandschaften zeigten Grundwasserdaten signifikante Zusammenhänge zwischen Cadmium und erhöhten Nitratkonzentrationen sowie Versauerung. Hohe Grundwasserneubildungsraten begünstigten eine direkte Beeinflussung des Grundwassers durch ackerbauliche und forstwirtschaftliche Nutzung, sodass eine erhöhte Lösung von Cadmium eintrat.

Messstellen mit steigenden Cadmiumkonzentrationen konnten über Trendtests ermittelt werden. Geestgebiete mit erhöhten Cadmiumkonzentrationen zeigten eine gute räumliche Übereinstimmung mit der Maßnahmenkulisse zur Nitratreduktion. Für hydrogeologische Teilräume wurden Cadmium-Hintergrundkonzentrationen zwischen $0,01 \mu \mathrm{g} / \mathrm{l}$ und $0,98 \mu \mathrm{g} / \mathrm{l}$ berechnet. Diese regional differenzierten Hintergrundwerte erlauben eine Neubewertung des chemischen Zustands der Grundwasserkörper.

Schlüsselwörter Cadmium · Grundwasser · Niedersachsen · Wasserrahmenrichtlinie

Andreas Kubier

a.kubier@uni-bremen.de

Dörte Budziak

doerte.budziak@lbeg.niedersachsen.de

Dieter de Vries

dieter.devries@nlwkn-aur.niedersachsen.de

Jörg Elbracht

joerg.elbracht@lbeg.niedersachsen.de

Kay Hamer

khamer@uni-bremen.de

Thomas Pichler

pichler@uni-bremen.de

1 Fachgebiet Geochemie und Hydrogeologie, Fachbereich Geowissenschaften, Universität Bremen, Postfach 330440, 28334 Bremen, Deutschland

2 Referat Hydrogeologische Grundlagen, Landesamt für Bergbau, Energie und Geologie (LBEG), Stilleweg 2, 30655 Hannover, Deutschland

3 Betriebsstelle Aurich, Niedersächsischer Landesbetrieb für Wasserwirtschaft, Küsten- und Naturschutz (NLWKN), Oldersumer Straße 48, 26603 Aurich, Deutschland 


\title{
Cadmium in groundwater of Northwestern Germany-origin, mobilization and assessment according to the European Water Framework Directive
}

\begin{abstract}
Nine groundwater bodies in northwestern Germany had a "bad" status according to the Water Framework Directive due to cadmium concentrations above $0.5 \mu \mathrm{g} / \mathrm{l}$. Data from fertilizer, soil, and groundwater analyses from statewide monitoring were used to study geogenic input, fertilizer emissions, and atmospheric deposition.

Until 2016, cadmium input decreased to $0.74 \mathrm{~g} /(\mathrm{ha} \cdot \mathrm{a})$ from phosphate fertilizers and $0.2 \mathrm{~g} /(\mathrm{ha} \cdot \mathrm{a})$ from atmospheric deposition. Groundwater data revealed a significant correlation between cadmium and nitrate plus acidification mainly in the agriculture-intensive Geesten areas. High groundwater recharge rates promote a direct impact on groundwater in agricultural and forestry areas causing increased cadmium solubility.

Time series analysis identified increasing cadmium trends and showed that Geesten areas with elevated cadmium concentrations in groundwater corresponded to areas where nitrate reduction measures are needed.

Cadmium background levels in groundwater ranged from $0.01 \mu \mathrm{g} / \mathrm{l}$ to $0.98 \mu \mathrm{g} / \mathrm{l}$. Using the better differentiated background levels allowed a re-evaluation of the chemical status of groundwater bodies.
\end{abstract}

Keywords Cadmium · Groundwater · Lower Saxony · Water Framework Directive

\section{Zielsetzung des Projekts}

Mit der Verabschiedung der EU-Wasserrahmenrichtlinie (WRRL) haben sich die Mitgliedsstaaten verpflichtet, Umweltziele für ihre Gewässer zu erreichen (RL 2000/60/EG). $\mathrm{Zu}$ den Voraussetzungen dafür zählen ein flächendeckendes repräsentatives Monitoring und die regelmäßige Bewertung der Ergebnisse. Bei Verfehlen der Umweltziele, im Grundwasser spricht man vom guten mengenmäßigen und chemischen Zustand, gilt es, geeignete Maßnahmen durchzuführen, die dann später diese Umweltziele erreichen lassen. Als Kriterien für den guten qualitativen Zustand der Grundwasserkörper (GWK) gelten Konzentrationen im Grundwasser für Stoffe, die in der Grundwasser-Tochterrichtlinie (RL 2006/118/EG) genannt sind. In Deutschland wurden diese europäischen Vorgaben in der Grundwasserverordnung konkretisiert und dort u. a. Schwellenwerte festgeschrieben (GrwV 2017; Anlage 2). Der Schwellenwert für Cadmium (Cd) liegt bei $0,5 \mu \mathrm{g} / 1$ (GrwV 2017). Dieser wurde zusammen mit abgeleiteten Hintergrundwerten für die Bewertung der GWK in Niedersachsen und Bremen herangezogen. Bei 9 von 123 GWK wurde keine plausible Begründung für erhöhte Cd-Konzentrationen oberhalb des Schwellenwertes oder alternativ zu den bestimmten Hintergrundwerte gefunden, sodass der chemische Zustand bei der Beurteilung nach WRRL in diesen Gebieten als schlecht eingestuft worden ist (MU 2015a, 2018).

Die grundsätzliche Frage war, ob das Cd geogen vorhanden ist oder ob anthropogene Einflüsse zum Eintrag in das Grundwasser bzw. zur Mobilisierung des Cd im Grundwasserleiter geführt haben. Zur Klärung der Frage, welche Ursachen und Einflüsse zu den erhöhten Cd-Konzentrationen im Grundwasser führen, wurden neben Grundwasserana- lysen der Landesmessnetze Niedersachsens und Bremens auch Analysen von atmosphärischer Deposition, Sickerwasser und Boden an den Boden-Dauerbeobachtungsflächen (BDF) in Niedersachsen (z.B. Höper und Meesenburg 2012) herangezogen. Zusätzlich wurden Literaturdaten zur chemischen Zusammensetzung eingesetzter Düngemittel recherchiert. Mit diesem Pool an Daten sollten Gebiete identifiziert werden, die bereits jetzt erhöhte Cd-Konzentrationen im Grundwasser aufweisen sowie Gebiete, in denen es möglicherweise in Zukunft zu erhöhten Cd-Werten im Grundwasser kommen kann. Für diese Gebiete ließen sich bei Kenntnis der beteiligten Prozesse, die die Mobilität von $\mathrm{Cd}$ beeinflussen, dann Maßnahmenfelder benennen, die zukünftig zur Reduzierung der Cd-Konzentrationen führen oder der Gefahr einer Mobilisierung entgegenwirken können.

\section{Forschungsstand}

Die Grundwasserneubildung findet über das Sickerwasser statt, das oft durch landwirtschaftliche Nutzung des Bodens beeinflusst ist. So kann z. B. Nitrat eingetragen und damit das hydrochemische Milieu verändert werden (Böhlke 2002). Schon Kunkel et al. (2004) erklärten daher, dass in Deutschland natürliches, von Menschen unbeeinflusstes oberflächennahes Grundwasser seit Jahrhunderten nicht mehr vorhanden ist, da die Vielfalt der landwirtschaftlichen Nutzungen wie Rodung, Ackerbau, und Melioration die hydrochemischen Verhältnisse flächendeckend und diffus beeinflusst haben. Je nach Landnutzung gelangen Einträge in das Grundwasser und führen zu einem ubiquitär überprägten Grundwasser. 
Durch die fortschreitende Oxidation über die Nitratverlagerung in tiefere Schichten wird das hydrochemische Verhalten redoxsensitiver Stoffe verändert. Dazu zählt indirekt auch $\mathrm{Cd}$, welches in Sulfiden gebunden sein kann, sodass beim Vorrücken der Denitrifikationszone das Cd zunächst gelöst wird. Die Konzentration im Grundwasser erhöht sich unabhängig davon, ob $\mathrm{Cd}$ durch $\mathrm{pH}$-Wertänderungen von Mineraloberflächen desorbiert oder als Bestandteil von Sulfidmineralen freigesetzt wird (Böhlke 2002; Cremer 2002), sodass dessen Konzentration im Grundwasser an die autotrophe Denitrifikation gekoppelt ist (Gl. 1).

$$
\begin{aligned}
& 5\left(\mathrm{Fe}_{1-\mathrm{x}} \mathrm{Cd}_{x}\right) \mathrm{S}_{2(\mathrm{~s})}+15 \mathrm{NO}_{3}^{-}+5 \mathrm{H}_{2} \mathrm{O} \rightarrow \\
& 5(1-x) \mathrm{FeOOH}+5 x \mathrm{Cd}^{2+}+7,5 \mathrm{~N}_{2(\mathrm{~g})}+10 \mathrm{SO}_{4}^{2-}+5 \mathrm{H}^{+}
\end{aligned}
$$

Pyrit ist neben organischem Material ein wichtiger Elektronendonator für den Nitratabbau (Denitrifikation) im anoxischen Grundwasser (Hinsby et al. 2008; Jorgensen et al. 2009). Bergmann et al. (2013) erläuterten, wie hoch der Anteil an Pyrit und organischer Substanz im Grundwasserleiter ist und in welchem Umfang beide das Nitratabbauvermögen am jeweiligen Standort darstellen. Die Denitrifikation puffert dabei Nitrateinträge in das Grundwasser. Indikatoren wie erhöhte Sulfatkonzentrationen konnten nur teilweise festgestellt werden, da Sulfat anthropogen und durch Lösungsprozesse eingetragen wird (Böhlke 2002). Auch andere Begleitstoffe in Pyrit wie z.B. Zn und Co sind nicht zwangsläufig im Grundwasser erhöht, da sie erst bei geringeren $\mathrm{pH}-$ Werten gelöst vorliegen und eher als $\mathrm{Cd}$ adsorbieren (Herms und Brümmer 1984). Im Emsland untersuchte Pyrite in reduzierten Grundwasserleitern wiesen Cd-Gehalte im Mittel von $300 \mathrm{mg} / \mathrm{kg}$, im Maximum von $1600 \mathrm{mg} / \mathrm{kg}$ und, je nach Pyritgehalt im Grundwasserleiter, bis $2,6 \mathrm{mg} / \mathrm{kg}$ im Sediment auf (Houben et al. 2017). Durch eine unterschiedliche Zusammensetzung der Sulfidminerale im Grundwasserleiter zeigt sich die an die Denitrifikation gekoppelte Pyritoxidation in erhöhten Konzentrationen von $\mathrm{Ni}, \mathrm{Co}, \mathrm{Zn}$ und As in der Niederrheinischen Bucht (Cremer 2002) sowie in erhöhten Urankonzentrationen in BadenWürttemberg (Riedel und Kübeck 2018) und MecklenburgVorpommern (van Berk und Fu 2017).

Der pH-Wert und das Redoxpotenzial haben wesentlichen Einfluss auf die Cd-Mobilität (z. B. Carrillo-Gonzalez et al. 2006; Krishnamurti und Naidu 2003). Diese wird über anthropogene Einträge in Form von Nitrat oder Säuren erhöht. Die Cd-Festlegung und Cd-Freisetzung in Böden wird somit durch Landnutzung, Substratart und die Grundwasserbeschaffenheit reguliert. Da die Geesten insbesondere im Westen Niedersachsens Regionen mit intensiver Landwirtschaft sind, lassen sich die erhöhten Cd-Konzentrationen mit dem dort auftretenden landwirtschaftlich geprägten, versauerten Grundwasser in Verbindung bringen (Wriedt et al. 2019).

Neben der Hydrochemie beeinflussen auch andere Faktoren den Eintrag sowie die Bindung von Cd im Untergrund. Schilli et al. (2011) untersuchten dazu den Zusammenhang zwischen Landnutzung und Bodenart auf die Mobilität von

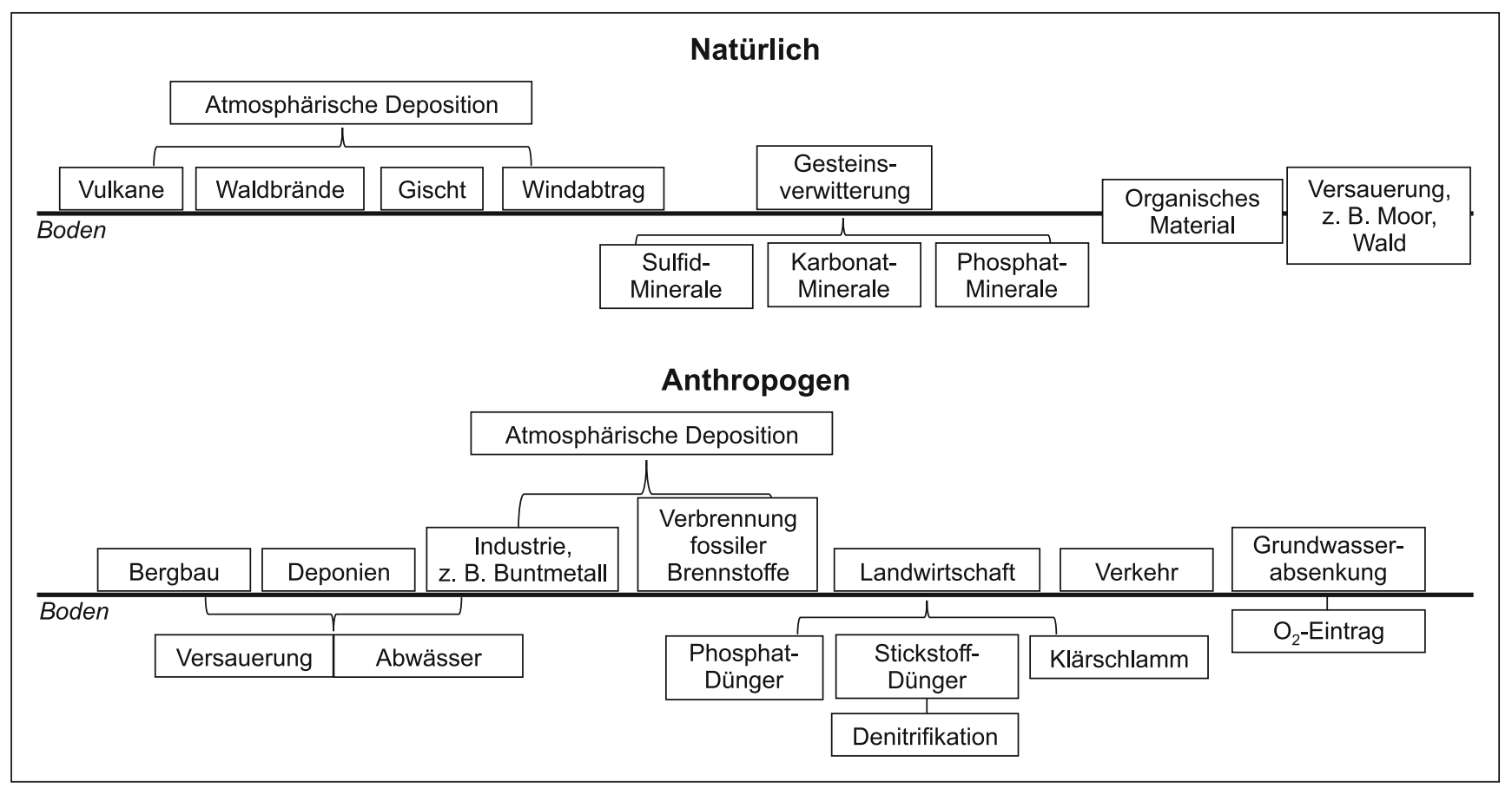

Abb. 1 Schema der natürlichen und anthropogenen Cadmium-Einträge bzw. Einflüsse auf die Freisetzung von Cadmium ins Grundwasser Fig. 1 Natural and anthropogenic cadmium sources and influences on Cd release into groundwater 
Tab. 1 Szenarien der Cadmium-Freisetzung bzw. -Eintragung

Table 1 Scenarios of cadmium release and contamination

\begin{tabular}{|c|c|c|c|}
\hline Szenario & $\begin{array}{l}\text { I: Geogener Ursprung, natürliche } \\
\text { Mobilisierung }\end{array}$ & $\begin{array}{l}\text { II: Geogener Ursprung, anthro- } \\
\text { pogen induzierte Mobilisierung }\end{array}$ & III: Anthropogener Eintrag \\
\hline Cd-Herkunft & $\begin{array}{l}\text { Minerale (Sulfide, Karbonate, } \\
\text { Hydr-/Oxide, Tone), organisches } \\
\text { Material }\end{array}$ & $\begin{array}{l}\text { Minerale (Sulfide, Karbonate, } \\
\text { Hydr-/Oxide, Tone), organisches } \\
\text { Material }\end{array}$ & $\begin{array}{l}\text { Phosphat-Dünger, atmosphäri- } \\
\text { sche Deposition, Punktquellen } \\
\text { (z. B. Abwasser, Deponie) }\end{array}$ \\
\hline Cd-Freisetzung & $\begin{array}{l}\text { Lösung aus Mineralen, Desorp- } \\
\text { tion von Hydr-/Oxiden, Tonen } \\
\text { oder organischem Material }\end{array}$ & $\begin{array}{l}\text { Lösung aus Mineralen, Desorp- } \\
\text { tion von Hydr-/Oxiden, Tonen } \\
\text { oder organischem Material }\end{array}$ & $\begin{array}{l}\text { Lösung im Boden, Verlagerung } \\
\text { mit Sickerwasser }\end{array}$ \\
\hline Ursache der Freisetzung & $\begin{array}{l}\text { Versauerung (Wald, Moor), sai- } \\
\text { sonal schwankender Grundwas- } \\
\text { serstand }\end{array}$ & $\begin{array}{l}\text { Eintrag von Säuren, Nitrat, Salz, } \\
\text { „Liganden“; Grundwasserabsen- } \\
\text { kung }\end{array}$ & Versickerung ins Grundwasser \\
\hline \multicolumn{4}{|l|}{ Schema } \\
\hline \multicolumn{4}{|l|}{ Boden } \\
\hline \multicolumn{4}{|l|}{ Grundwasserleiter } \\
\hline Sickerwasser & $\mathrm{Cd}^{2+}$ & $\mathrm{Cd}^{2+}$ & $\mathrm{Cd}^{2+}$ \\
\hline Anthropogen gelöst & & & \\
\hline Anthropogen fest & & & \\
\hline Freisetzung & $\mathrm{Cd}^{2+}$ & $\mathrm{Cd}^{2+}$ & \\
\hline
\end{tabular}

mehreren Schwermetallen. Sie stellten fest, dass weitere Faktoren dafür sorgen, dass die Cd-Gehalte im Boden im Gegensatz zu den anderen untersuchten Schwermetallen $\mathrm{Cr}$, $\mathrm{Ni}, \mathrm{Cu}, \mathrm{Zn}$ und $\mathrm{Pb}$ nicht primär durch das Ausgangsgestein der Bodenbildung erklärt werden konnten. Neben der Substratart und dem pH-Wert beeinflussen auch der Auskämmeffekt und der organische Horizont an Forststandorten, Sonderfälle wie der Bereich des durch Bergbau beeinflussten Harzes sowie Einträge durch atmosphärische Deposition und Mineraldünger den Cd-Gehalt des Bodens (Schilli et al. 2011).

Mögliche Quellen des Cd und die bei der Mobilisierung beteiligten Prozesse waren Gegenstand des Forschungsprojektes „Cadmium im Grundwasser Niedersachsens“ (Kubier et al. 2018), welches als Maßnahme nach WRRL initiiert wurde (MU 2015b). Quellen und Faktoren, die die Cd-Konzentration im Grundwasser beeinflussen können, lassen sich in oberirdische und unterirdische sowie in natürliche und anthropogene Faktoren einteilen (Abb. 1). Im Falle natürlicher Ursachen kann eine Hintergrundkonzentration für $\mathrm{Cd}$ im Grundwasser abgeleitet werden, die, falls sie oberhalb des Schwellenwertes von 0,5 $\mu \mathrm{g} / \mathrm{l}$ liegt, anstatt des Schwellenwertes für das betroffene Gebiet als Bewertungskriterium für den chemischen Zustand von GWK herangezogen wird (GrwV 2017). Als anthropogene Ursachen sind hingegen der Eintrag von $\mathrm{Cd}$ aus der Atmosphäre oder als Nebenbestandteil von Phosphat-Düngern genauso denkbar wie die Mobilisierung von im Sediment vorhandenem $\mathrm{Cd}$ in das Grundwasser.

In Tab. 1 sind drei Szenarien zur Cd-Herkunft im Grundwasser erläutert und gegenübergestellt.

\section{Untersuchungsgebiet}

Der Großteil Niedersachsens und Bremens ist geologisch durch das Pleistozän geprägt. An der Küste liegen fluviatile Gezeitenablagerungen vor, weiter nach Süden finden sich Schmelzwasserablagerungen, Flussablagerungen oder Moore. Südlich der Linie Nordhorn - Hannover - Wolfsburg schließt sich das Mitteldeutsche Bruchschollenland an, wobei auch dort verbreitet quartäre Ablagerungen an der Geländeoberfläche auftreten. Schließlich ist im Südosten der Harz als Teil des Mitteldeutschen Grundgebirges anzutreffen (Elbracht et al. 2016).

Die Bezugsflächen zur Beurteilung der Grundwasserqualität nach WRRL sind die Grundwasserkörper (GWK) (MU 2015a, 2018). Niedersachsen und Bremen sind demnach in 123 GWK unterteilt. Diese orientieren sich an den Teileinzugsgebieten der Oberflächengewässer. Da diese nach hydraulischen Kriterien zugeschnitten sind und nicht unbedingt geologische noch hydrochemische Eigenheiten im Grundwasserleiter abbilden, wurde im Rahmen der vorliegenden Studie die hydrogeologische Gliederung in Groß- 


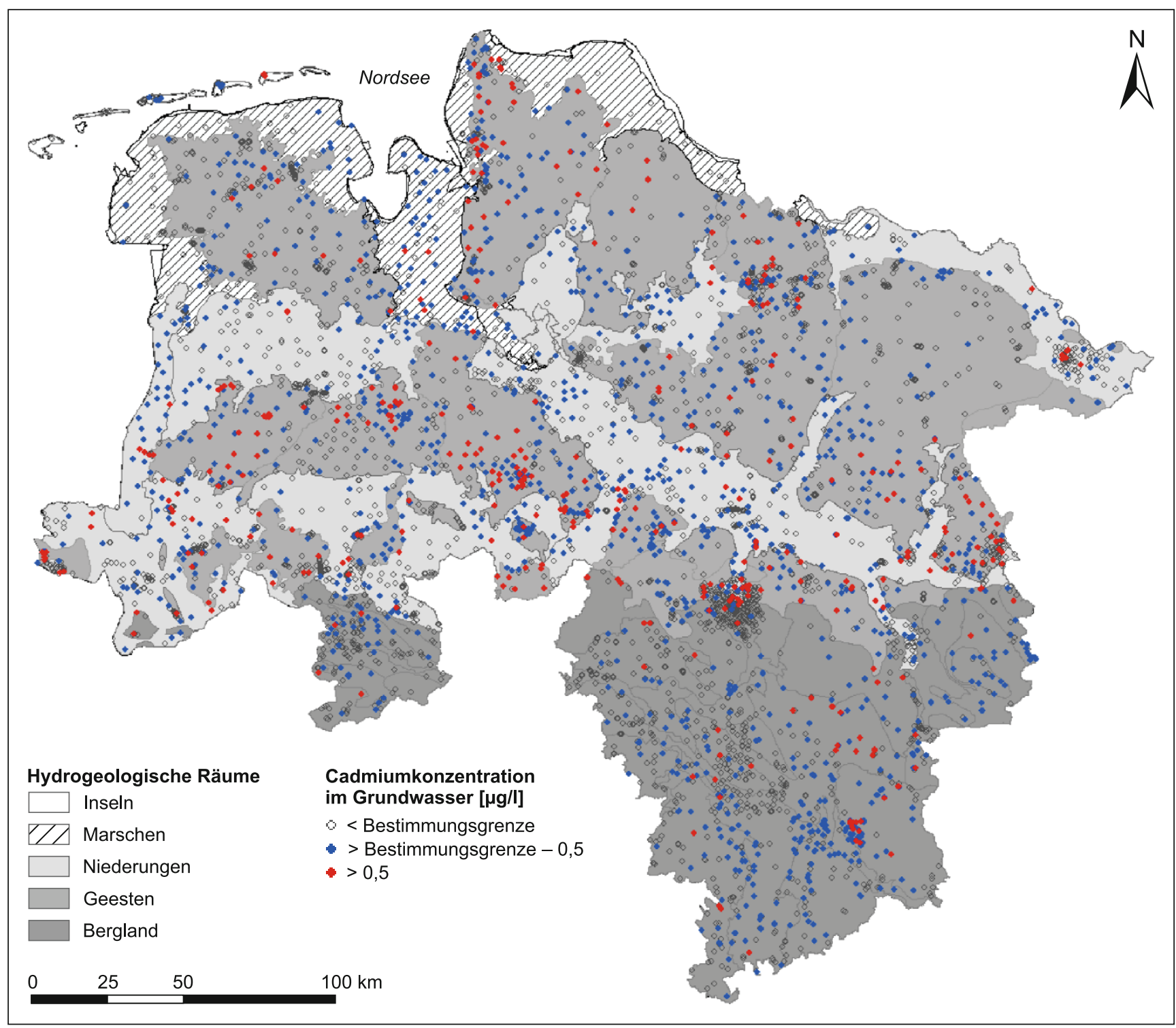

Abb. 2 Cadmiumkonzentrationen im Grundwasser Niedersachsens und Bremens entsprechend der Hydrogeologischen Teilräume (Kubier und Pichler 2019, verändert)

Fig. 2 Cadmium concentrations in groundwater of Lower Saxony and Bremen according to the hydrogeological subareas (Kubier and Pichler 2019, modified)

räume, Räume und Teilräume verwendet (Abb. 2), die im Gegensatz zu den GWK hydrogeologisch abgeleitet sind (Elbracht et al. 2016). Die Geesten unterscheiden sich dabei im Vergleich zu den Niederungen insbesondere durch ihr Relief und ihre geologische Entstehung. Hydrogeologische Merkmale gegenüber den Niederungen sind größere Flurabstände, eine hohe Grundwasserneubildung und ein teils geringes Schutzpotenzial der Deckschichten für das Grundwasser (Ad-hoc-Arbeitsgruppe Hydrogeologie 2016; Elbracht et al. 2016). Im Untersuchungsgebiet ist die Landnutzung überwiegend durch die Landwirtschaft geprägt, wobei die Hälfte der Fläche als Ackerland, ein Fünftel als
Grünland und ein weiteres Fünftel als Wald genutzt wird (LBM-DE 2012).

\section{Methodik}

\section{Datensatz}

Niedersachsen und Bremen werden flächendeckend mit Grundwasser-Messstellen erfasst (Abb. 2). Grundwasseranalysen aus Niedersachsen wurden bereitgestellt durch das Landesamt für Bergbau, Energie und Geologie (LBEG), den Niedersächsischen Landesbetrieb für Wasserwirtschaft, 
Küsten- und Naturschutz (NLWKN 2014) und die Landeshauptstadt Hannover sowie aus Bremen durch den Senator für Umwelt, Bau und Verkehr (SUBV). Der im Projekt genutzte Datensatz beider Bundesländer umfasste 6275 Grundwasser-Messstellen mit insgesamt 25.782 Analysen aus einem Zeitraum von 1976 bis 2016. Der Datensatz wurde auch in anderen Veröffentlichungen genutzt (Kubier und Pichler 2019; Kubier et al. 2020), sodass es teilweise zu inhaltlichen Überschneidungen kommt. Diese sind jedoch für ein besseres Verständnis des Zusammenhangs zwischen Wasserrahmenrichtlinie und entsprechenden Maßnahmen der zuständigen Behörden erforderlich. Darüber hinaus werden hier erstmalig Daten gezeigt und besprochen, die vorher nicht Gegenstand waren. Dies betrifft die Bodenanalysen aus den Boden-Dauerbeobachtungsflächen (BDF), die Verknüpfung der chemischen Analysen des Grundwassers mit dem Grundwasserleiter, die Umsetzung der Wasserrahmenrichtlinie auf regionaler Basis mit Ergebnissen, die zu einer Neubewertung der Daten führen werden, sowie die Thematik der Schwellenwerte allgemein.

Die Hälfte der Messstellen im Datensatz wurde nur einmal auf $\mathrm{Cd}$ analysiert. Demgegenüber standen vereinzelte Messstellen mit bis zu $44 \mathrm{Cd}$-Werten. Die meisten untersuchten Wässer stammten aus Tiefen bis zu $20 \mathrm{~m}$ in den Niederungen bzw. $60 \mathrm{~m}$ auf der Geest unter Geländeoberkante. An 737 Messstellen bzw. 2147 Analysen wurde der Geringfügigkeitsschwellenwert von $0,5 \mu \mathrm{g} / \mathrm{l} \mathrm{Cd}$ überschritten. Ein Blick auf die Filtertiefen der Messstellen zeigt, dass $\mathrm{Cd}>0,5 \mu \mathrm{g} / \mathrm{l}$ im flacheren, oberflächennahen Grundwasser erhöht auftrat (Abb. 3).

Zur Qualitätssicherung wurde für jede Grundwasseranalyse die Ionenbilanz berechnet. Zusätzlich $\mathrm{zu}$ den hydrochemischen Daten, bestehend aus Vor-Ort-Parame-

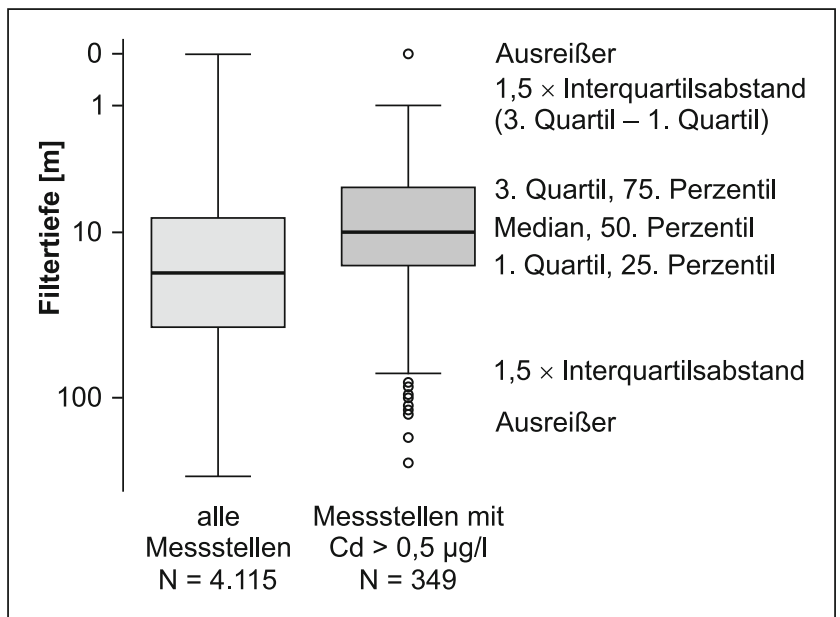

Abb. 3 Boxplots der Filtertiefe aller Messstellen (links) und der Messstellen mit $\mathrm{Cd}>0,5 \mu \mathrm{g} / \mathrm{l}$ (rechts). Die Tiefenangabe ist logarithmisch dargestellt

Fig. 3 Boxplots of screen depth from all wells (left) and wells with $\mathrm{Cd}>0.5 \mu \mathrm{g} / \mathrm{l}$. Depth is displayed logarithmically tern, Hauptparametern und Spurenstoffen, wurden auch die Stammdaten wie Koordinaten oder Filtertiefe und der Wasserstand der Grundwasser-Messstellen abgefragt. Der Datensatz wurde mit ArcGIS (ESRI 2018) graphisch projiziert. Über das Verschneiden mit topographischen, geologischen und hydrogeologischen Karten wurde der Datensatz um folgende Attribute ergänzt: Landnutzung (LBM-DE2012), geologische Einheit (LBEG 2000), hydrogeologische Einheit (LBEG 2004), Schutzpotenzial der Deckschichten, Grundwasseroberfläche und -neubildung (LBEG 2008, 2015).

Neben den Grundwasseranalysen wurden auch Daten zur Deposition, Bodenbeschaffenheit und Sickerwasser von 81 Boden-Dauerbeobachtungsflächen (BDF) ausgewertet. Die Analysen von landwirtschaftlich genutzten BDF (BDFL) stammten vom LBEG sowie NLWKN und Daten vom Forst-BDF (BDF-F) wurden von der Nordwestdeutschen Forstlichen Versuchsanstalt (NW-FVA) Göttingen geliefert. An 22 sogenannten Intensiv-BDF (BDF-I) werden zudem regelmäßig das Sickerwasser und teilweise auch die atmosphärische Deposition beprobt und analysiert (Höper und Meesenburg 2012).

\section{Auswertung}

Es wurden räumliche und zeitliche Verteilungen der CdKonzentrationen im Grundwasser ermittelt. Zusätzlich wurden mit dem Tendenz-Test nach Mann-Kendall geprüft, ob die zeitliche Entwicklung der Cd-Konzentrationen an einzelnen Messstellen einen signifikanten $(p<0,05)$ Verlauf aufwies. Bei 2223 Grundwasser-Messstellen lagen mindestens vier Cd-Analysen mit wenigstens einer Analyse $\mathrm{Cd}>0,5 \mu \mathrm{g} / \mathrm{l}$ vor, sodass hier ein Trendtest nach MannKendall vorgenommen werden konnte.

Der Mann-Kendall-Test (Kendall 1975; Mann 1945) erlaubt auch bei begrenzter Datendichte und unregelmäBig beprobten Datenzeiträumen die Feststellung, ob ein Trend innerhalb der jeweiligen Datenreihe vorliegt. Der Test ist ein rangstatistisches, verteilungsfreies Testverfahren, in dem zunächst zwei Hypothesen erstellt werden: Eine Nullhypothese, die besagt, dass kein Trend vorliegt und eine Gegenhypothese, die besagt, dass es einen Trend gibt. Der Test prüft das Eintreten der Nullhypothese. Dazu wird durch systematisches Vergleichen der einzelnen Messdaten miteinander eine Datenmatrix erstellt, in der die relativen Magnituden der verglichenen Messdaten jeweils mit einem Wert von ,, +1 “ für steigend und , -1 “ für fallend eingetragen werden. Die Werte werden summiert und als Ergebnis ein positiver, negativer oder uneindeutiger Trend festgestellt (Aziz et al. 2004).

Aus der weiteren Auswertung wurden solche Messstellen entfernt, die nur vereinzelte oder nicht plausible erhöhte Cd-Konzentrationen aufwiesen. 
Für die weitere Einschätzung der Cd-Konzentrationen wurden der natürliche vom wahrscheinlich anthropogen beeinflussten Daten-Anteil mithilfe des Wahrscheinlichkeitsnetzes getrennt. Das Wahrscheinlichkeitsnetz ist ein graphisches Verfahren nach Lepeltier (1969) zur Überprüfung von Konzentrationsverteilungen eines Datensatzes. Es folgt der Annahme, dass die meisten Elemente (log-)normal verteilt sind. Die lognormalverteilten Abschnitte im Wahrscheinlichkeitsnetz erscheinen bei logarithmischer Skalierung als Gerade. Die Vermischung von Normal- und Teilpopulationen ist anhand von Teilgeraden unterschiedlicher Steigung durch die logarithmische Darstellung erkennbar. Nach dieser Datendarstellung ergibt sich der geogene Hintergrundwert als das 90. Perzentil der Normalpopulation (Wagner et al. 2011). Für die Hintergrundwerte wurde die jeweils zuletzt gemessene Analyse nur an den Messstellen berücksichtigt, die Cd-Konzentrationsverläufe ohne Trend aufwiesen. Zudem wurden nur Analysen berücksichtigt, bei denen die Bestimmungsgrenze $<1 \mu \mathrm{g} / \mathrm{l} \mathrm{Cd}$ betrug.

Zwischen verschiedenen Parametern der Grundwasseranalysen (Tab. 2) wurde eine Rangkorrelation nach Spearman (Spearman 1904) durchgeführt, die als statistisches Argument zur späteren Interpretation von Abhängigkeiten der Parameter oder Attribute untereinander genutzt wurden. Der Korrelationskoeffizient $r$ stellt dabei das Maß für die Güte eines linearen Zusammenhangs dar und nimmt

Tab. 2 Korrelationskoeffizienten der Rangkorrelation nach Spearman für Cd. Die Korrelationen sind auf dem Niveau von 0,01 (2-seitig) signifikant (Kubier und Pichler 2019, verändert)

Table 2 Spearman's rank correlation coefficients for Cd. Correlations had a two-tailed significance at the 0.01 level (Kubier and Pichler 2019, modified)

\begin{tabular}{ll}
\hline Parameter & Korrelationskoeffizient \\
\hline $\mathrm{Sb}$ & 0,66 \\
$\mathrm{~Pb}$ & 0,62 \\
$\mathrm{Cu}$ & 0,59 \\
$\mathrm{Co}$ & 0,52 \\
$\mathrm{Tl}$ & 0,52 \\
$\mathrm{Ni}$ & 0,51 \\
$\mathrm{Zn}$ & 0,49 \\
$\mathrm{Hg}$ & 0,44 \\
$\mathrm{U}$ & 0,39 \\
$\mathrm{NO}$ & 0,37 \\
$\mathrm{Cr}$ & 0,36 \\
$\mathrm{Se}$ & 0,33 \\
$\mathrm{O}_{2}$ & 0,26 \\
$\mathrm{SiO}$ & $-0,41$ \\
$\mathrm{PO}_{4}$ & $-0,38$ \\
$\mathrm{HCO}_{3}$ & $-0,23$ \\
$\mathrm{Fe}$ & $-0,22$ \\
$\mathrm{NH}_{4}$ & $-0,22$ \\
$\mathrm{pH}$ & $-0,2$ \\
\hline
\end{tabular}

Werte von $r= \pm 1$ bei einem vollkommenen Zusammenhang und $\mathrm{r}=0$ bei vollkommener Unabhängigkeit an. Das Vorzeichen bestimmt die Richtung des Zusammenhangs. Diese Berechnung setzt keine Normalverteilung voraus und eine statistische Signifikanz der Korrelationsergebnisse wird ab einem $p$-Wert mit 0,01 angenommen (siehe auch Kubier und Pichler 2019).

Jährliche Cd-Frachten für die atmosphärische Deposition wurden aus den 14-tägigen Depositionsdaten im Rahmen des Monitorings zu den Bodendauerbeobachtungsflächen (BDF) berechnet (Keuffel-Türk et al. 2012). Zusätzlich wurden die $\mathrm{Cd}$-Gehalte in den Bodenanalysen der BDF als Königswasseraufschluss sowie als EDTA-Extrakt ausgewertet, wobei der Anteil des EDTA-Extrakts bezogen auf den Königswasseraufschluss als der Anteil betrachtet wird, der in das Grundwasser gelöst werden kann. Daraus wird jeweils der Anteil des mobilisierbaren $\mathrm{Cd}$ im Boden und im Humus berechnet. An den BDF mit Schwermetallanalysen in der wässrigen Phase (BDF-I) wurden die Zeitreihen für $\mathrm{Cd}$ in Deposition, Sickerwasser und, wenn vorhanden, von Grundwasser-Messstellen ausgewertet. Informationen zur Beprobungstiefe, -häufigkeit und der Analytik sind Höper und Meesenburg (2012) zu entnehmen.

\section{Ergebnisse und Diskussion}

\section{Verteilung der Cadmiumkonzentrationen}

An 210 Grundwasser-Messstellen lagen Zeitreihen mit CdKonzentrationen $>0,5 \mu \mathrm{g} / \mathrm{l}$ vor. Davon hatten 30 Zeitreihen nach Mann-Kendall-Test einen steigenden Verlauf und 53 gleichbleibend hohe Cd-Konzentrationen. Auffällig war, dass Messstellen mit $\mathrm{N} \geq 4$ und $\mathrm{Cd}>0,5 \mu \mathrm{g} / \mathrm{l}$ überwiegend in den Teilräumen der Geest lagen, besonders in der Syker Geest (Abb. 4).

Ein exemplarischer Verlauf von Zeitreihen mit Daten des NLWKN (Abb. 5) zeigt, wie sich die Konzentrationen von $\mathrm{Cd}$ und Nitrat an der Messstellengruppe Hüven in der Sögeler Geest von 1987 bis 2014 entwickelten. Während im Bereich des tiefen Filters weder $\mathrm{Cd}$ noch Nitrat zu beobachten waren, war im flachen Grundwasser im Schnitt eine stetige und gleichförmige Zunahme bei beiden Parametern zu erkennen. Cd-Konzentrationen stiegen bis auf $4,8 \mu \mathrm{g} / \mathrm{l}$. Um zu überprüfen, ob ein statistisch signifikanter Zusammenhang zwischen Parametern bestand, wurden im Rahmen der Datenanalyse außerdem univariate Korrelationsanalysen durchgeführt. Die dabei errechneten Rangkorrelation nach Spearman (Tab. 2) zeigten zwischen $\mathrm{Cd}$ und anderen Schwermetallen starke Korrelationen (Korrelationskoeffizient r, z. B. mit $\mathrm{Pb} 0,62 ; \mathrm{Cu} 0,59 ; \mathrm{Co} 0,52 ; \mathrm{Ni}$ $0,51)$ und ergaben eine mittlere Korrelation zwischen $\mathrm{Cd}$ und Nitrat von $r=0,37$, was nach Cohen (1988) eine mitt- 


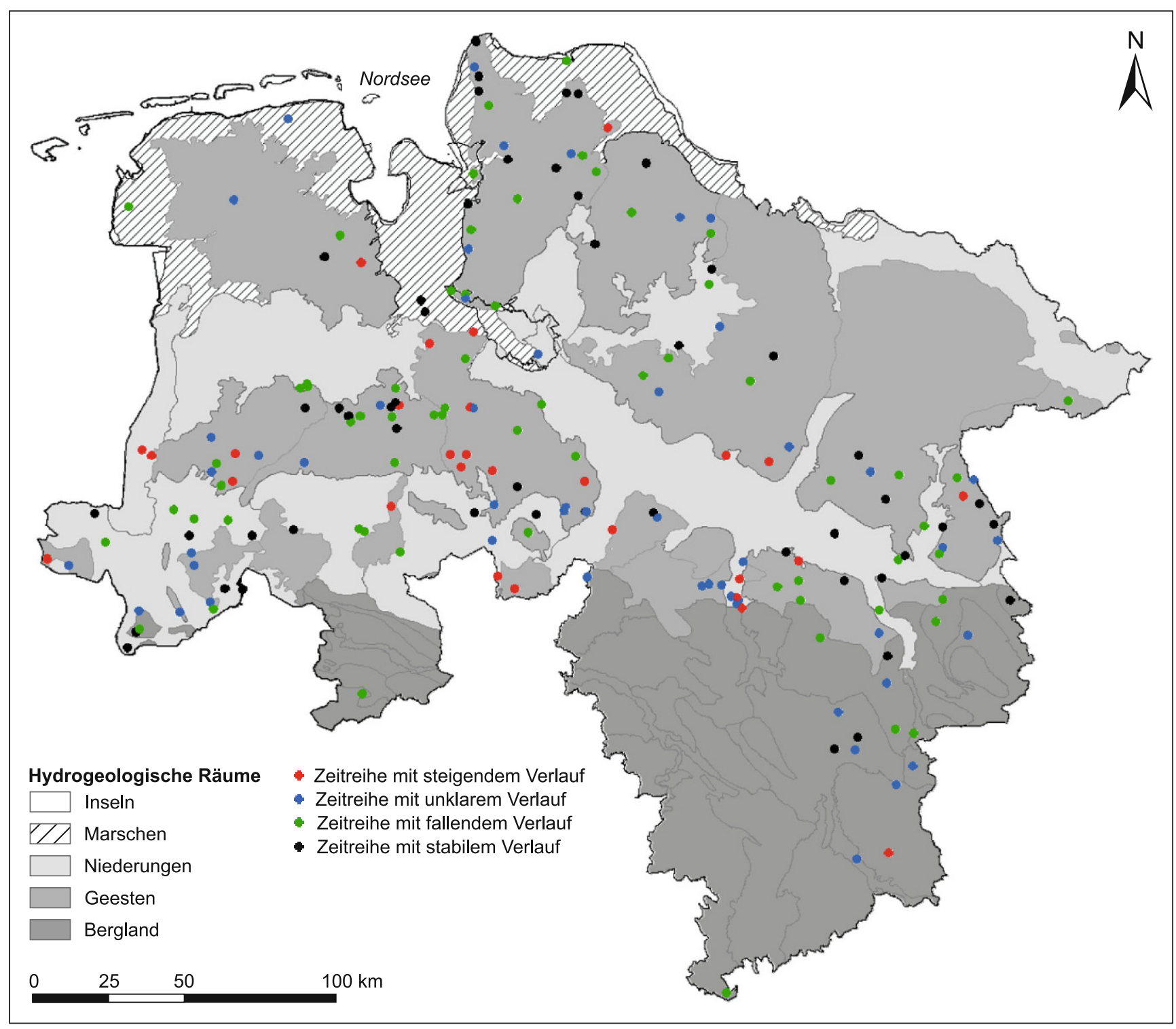

Abb. 4 Lage der Messstellen mit Cd-Zeitreihen in den Hydrogeologischen Teilräumen

Fig. 4 Location of $\mathrm{Cd}$ time series wells in the hydrogeological subareas

lere Korrelation darstellt. Negative Korrelationen wurden gefunden zwischen Cd und Parametern, die auf reduzierende oder alkalische Bedingungen hinweisen, wie z.B. mit $\mathrm{HCO}_{3}(\mathrm{r}=-0,23)$ und $\mathrm{Fe}(\mathrm{r}=-0,22)$. Die Korrelation mit dem $\mathrm{pH}-$ Wert war auch negativ $(\mathrm{r}=-0,22)$.

Bis auf wenige kleine Teilräume konnten flächendeckend Hintergrundwerte für $\mathrm{Cd}$ abgeleitet werden (Abb. $7 \mathrm{im} \mathrm{An-}$ hang; Kubier et al. 2020). Diese Werte schwanken zwischen $<0,2 \mu \mathrm{g} / \mathrm{l}$ und $>0,5 \mu \mathrm{g} / \mathrm{l}$. Die Hintergrundwerte in den Marschen und Niederungen lagen im Mittel bei $0,13 \mu \mathrm{g} / \mathrm{l}$. Im Gegensatz dazu wiesen die Grundwässer der Geesten meist erhöhte Werte auf, bei einem Mittelwert für Cd von $0,36 \mu \mathrm{g} / \mathrm{l}$. In der Syker Geest und der Bederkesa Geest erreichen sie sogar $>0,5 \mu \mathrm{g} / \mathrm{l} \mathrm{Cd}$. Das Grundwasser in den
Geesten wies generell höhere Hintergrundwerte für $\mathrm{Cd}$ als andere hydrogeologische Teilräume auf. Das Grundwasser hat dabei meist geringe $\mathrm{pH}$-Werte $(48 \%$ der Analysen aus den Geesten mit $\mathrm{pH}<6,5$, demgegenüber sind es $37 \%$ der Analysen im gesamten Datensatz) sowie ein oxisches Milieu (48\% der Analysen aus den Geesten mit Eh> $200 \mathrm{mV}$, demgegenüber sind es $39 \%$ im gesamten Datensatz). Beides sind Randbedingungen, die zu höherer Cd-Freisetzung von der Boden- oder Aquifermatrix führen und dann in diesen Gebieten mit hoher Grundwasserneubildungsrate auch zu höheren Cd-Konzentrationen im Grundwasser führen können (Carrillo-Gonzalez et al. 2006).

Demnach sind auch die hier abgeleiteten Hintergrundwerte für $\mathrm{Cd}$ im Grundwasser nicht unbedingt ein Indikator 


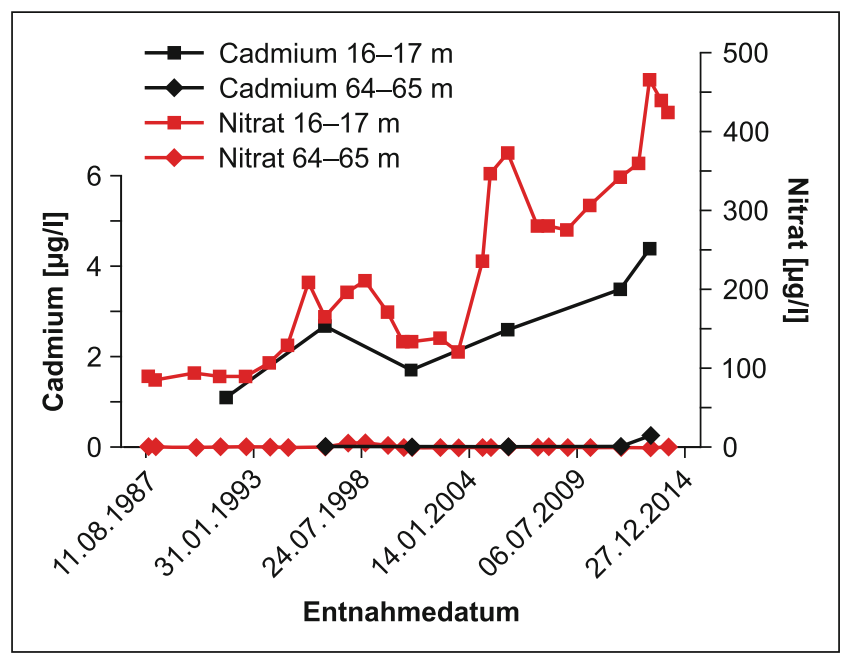

Abb. 5 Zeitreihen des NLWKN für Cadmium und Nitrat an der Messstellengruppe Hüven I (Filtertiefe 16-17 m) und Hüven II (Filtertiefe 64-65 m). Der Flurabstand beträgt ca. 7,7 m (Budziak et al. 2017, verändert)

Fig. 5 Time series from NLWKN of cadmium and nitrate at the well collective Hüven I (screen depth 16-17 m) and Hüven II (screen depth 64-65 m). Depth to water table is approx. $7.7 \mathrm{~m}$ (Budziak et al. 2017, modified)

für rein geogen bedingtes Cd. Denn ein weiterer Grund für die erhöhten Hintergrundwerte in den Geesten kann die mögliche Herkunft des $\mathrm{Cd}$ aus den eiszeitlich abgelagerten Gesteinen (Saale- und Weichsel-Kaltzeit) sein, welche dort als Grundwasserleiter dienen. Beim Kontakt, z. B. mit Nitrat, können Sulfidminerale gelöst werden (Koopmann et al. 2020) und das in Spuren in diesen Mineralen enthaltene Cd in das Grundwasser gelangen (Böhlke 2002). Einige Geesten haben ungewöhnlich geringe Hintergrundwerte mit $\mathrm{Cd}<0,1 \mu \mathrm{g} / \mathrm{l}$, die im Bereich der Hintergrundwerte der Marschen und vieler Niederungen liegen, was zeigt, dass diese Geesten als Moor-Geesten bzw. Übergang zum Bergland einen anderen hydrogeologischen und hydrochemischen Charakter haben (Kubier et al. 2020).

Die Ableitung von Hintergrundwerten für $\mathrm{Cd}$ für hydrogeologische Teilräume hat den Vorteil, mögliche Maßnahmen im wasserwirtschaftlichen Handeln auf besser zugeschnittene Kulissen begrenzen zu können, statt einen gesamten Grundwasserkörper als Gebiet für Maßnahmen zu sehen (siehe Abb. 7 im Anhang).

Von den insgesamt 6275 beprobten Grundwasser-Messstellen lag bei 364 Messstellen die jeweils zuletzt gemessene Cd-Konzentration über dem Wert von 0,5 $\mu \mathrm{g} / \mathrm{l}$. Die meisten dieser Messstellen befinden sich in Bereichen, in denen die Grundwässer überwiegend durch hohe Nitratkonzentrationen und Versauerung geprägt sind. Damit entsprechen lediglich $14 \%$ dieser 364 Messstellen $(N=52)$ dem Szenario I „Geogener Ursprung, natürliche Mobilisierung“ aus Tab. 1, während etwa drei Viertel der Messstellen $(N=265)$ dem Szenario II „Geogener Ursprung, anthropogen induzierte Mobilisierung“ oder III „Anthropogener Eintrag“ zuzuordnen sind. Daher kann davon ausgegangen werden, dass die erhöhten Cd-Konzentrationen häufig mit anthropogenen Aktivitäten in Verbindung stehen. Bei 47 Messstellen konnte keine eindeutige Zuordnung zu den drei Szenarien erfolgen.

Auf Grundlage dieser Punktinformationen, die um weitere Eigenschaften der betroffenen Standorte ergänzt wurden, konnten Kriterien und damit auch Gebiete für ein CdVerlagerungsrisiko abgeleitet werden. Neben der landwirtschaftlichen Beeinflussung und Versauerung sind Bereiche betroffen und damit als gefährdet einzustufen, die in den Teilräumen der Geesten liegen. Als typische Landnutzung wurden Acker und Wald ausgemacht (LBM-DE2012). Flache Filtertiefen bis zu $15 \mathrm{~m}$ unter GOK im Bereich von Grundwässern mit einem Flurabstand bis $10 \mathrm{~m}$ begünstigen ebenfalls erhöhte Cd-Konzentrationen. Die Gebiete mit CdVerlagerungsrisiko, die sich so ergeben, sind zum Großteil deckungsgleich mit der Maßnahmenkulisse Nitratreduktion (Abb. 6) des Landes Niedersachsen.

\section{Cadmiumeinträge und -freisetzung in das Grundwasser}

Neben der geogenen Konzentration von $\mathrm{Cd}$ im Boden und Grundwasser sind oberflächennah Einträge von $\mathrm{Cd}$ durch Deposition aus der Atmosphäre oder als Bestandteil von Düngemitteln, insbesondere Phosphat-Düngern, denkbar. Niederschlagswasser löst das $\mathrm{Cd}$ an der Bodenoberfläche, und über die Grundwasserneubildung wird es dann mit dem Sickerwasser ins Grundwasser transportiert.

Ausgehend von mittleren Cd-Gehalten im Boden zwischen $0,002 \mathrm{mg} / \mathrm{kg}$ und $1,8 \mathrm{mg} / \mathrm{kg}$ an den forstwirtschaftlich genutzten Flächen (BDF-F) und zwischen $0,001 \mathrm{mg} / \mathrm{kg}$ und $1,66 \mathrm{mg} / \mathrm{kg}$ an den landwirtschaftlich genutzten Standorten (BDF-L) zeigten die Cd-Gehalte des Bodens im ausgewerteten Datensatz abnehmende Gehalte in der Reihenfolge Grünland $>$ Acker $>$ Forst, was auch deutschlandweit nachgewiesen wurde (Huschek et al. 2004). Der Zusammenhang ergibt sich aus dem Zusammenspiel verschiedener Faktoren, welche die Mobilität des gelösten Cd beeinflussen. Beispielsweise ist der mittlere $\mathrm{pH}-$ Wert der Böden abnehmend in der Reihenfolge Acker $>$ Grünland $>$ Forst. Die Korngrößenverteilung der Böden zeigten, dass Standorte mit Grünland primär Ton, Moor und Lehm aufwiesen, während bei Acker und Forst eher Sand und Schluff mit deutlich geringerer Sorptionskapazität (Duijnisveld et al. 2008) auftraten.

Die Bindung von $\mathrm{Cd}$ im Boden ist an bestimmte Tiefen und Milieus gekoppelt. Die Analysen der BDF zeigten, dass sich mit zunehmender Tiefe die Cd-Gehalte des Bodens verringerten. Die Bindungsformen von Cd waren, wie auch von Krishnamurti und Naidu (2003) beobachtet, pH-abhängig. 


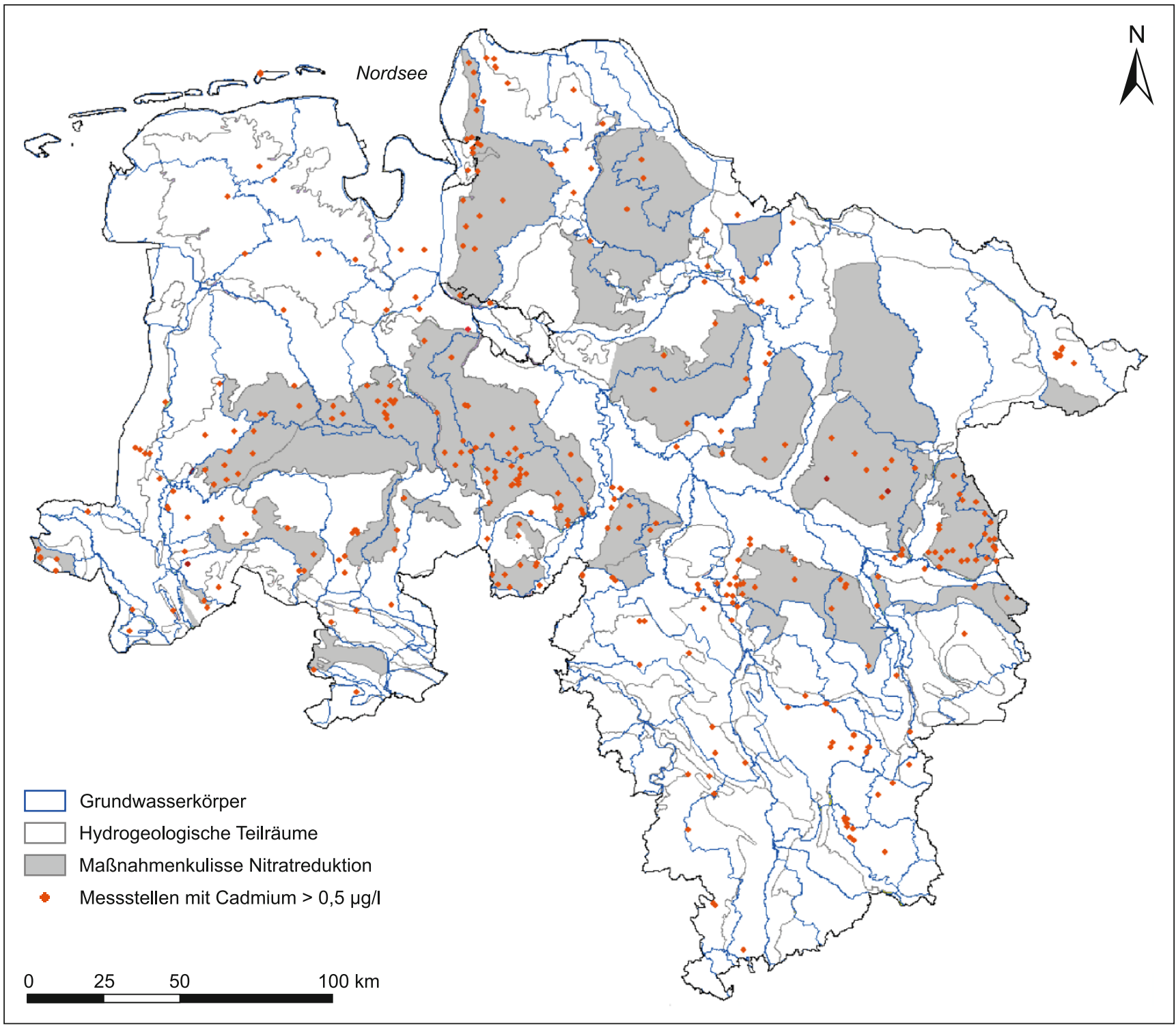

Abb. 6 Lage der Grundwasser-Messstellen mit Cadmium >0,5 $\mu \mathrm{g} / \mathrm{l}$ und Gebiete mit der Maßnahmenkulisse zur Nitratreduktion (MU 2015b, verändert)

Fig. 6 Well locations expressing cadmium $>0.5 \mu \mathrm{g} / \mathrm{l}$ and areas of the action planning to reduce nitrate in groundwater (MU 2015b, modified)

So stieg der Anteil des mobilisierbaren Cd (als EDTA-Extrakt) in den Bodenproben bei pH-Werten unter 4,5 deutlich an. Mit zunehmendem Zersetzungsgrad des Humus wurden bei den Analysen an den BDF neben geringer werdenden pH-Werten auch höhere Cd-Gehalte in den Humuslagen gemessen. Die erhöhten Cd-Gehalte im Boden sind somit das Resultat der auf ihn wirkenden Faktoren. Daraus lässt sich folgern, dass Standorte mit geringem $\mathrm{pH}$-Wert, sandigem Substrat und gesteigerten Einträgen durch Deposition im Zuge des Auskämmeffekts an Forststandorten oder Landwirtschaft mit Einsatz vom Phosphat-Dünger besonders geeignet sind, so eingetragenes $\mathrm{Cd}$ in das Sickerwasser abzugeben (Mollema et al. 2015).
Die Cd-Konzentration des Grundwassers hängt dann weiter von der Grundwasserneubildung, dem Flurabstand und der $\mathrm{pH}$-Wert-abhängigen Sorptionskapazität des Grundwasserleiters ab. Besonders in den Geesten sind diese Faktoren so kombiniert, dass hier die höheren Cd-Konzentrationen im Grundwasser von Niedersachsen und Bremen auftreten (Abb. 2). Messstellen mit erhöhten Cd-Konzentrationen liegen überwiegend in Bereichen mit einer Grundwasserneubildung von $150-250 \mathrm{~mm} / \mathrm{a}$. Demgegenüber hat der Großteil der Messstellen ohne auffällige Cd-Konzentrationen meist eine geringere Neubildung und dann häufig $\mathrm{im}$ Bereich $\leq 50 \mathrm{~mm} / \mathrm{a}$. Solche Randbedingungen sind notwendige Voraussetzungen für den direkten Eintrag von $\mathrm{Cd}$ 
Tab. 3 Cadmium-Frachten in Böden Nordwestdeutschlands

Table 3 Cadmium inputs and outputs in soils of Northwestern Germany

\begin{tabular}{|c|c|c|c|}
\hline & \multicolumn{3}{|c|}{ Cadmium-Fracht in g/(ha - a) (Min - Median - Max) } \\
\hline & $1980 \mathrm{er}$ bis $1990 \mathrm{er}$ & 2000er & 2010er \\
\hline \multicolumn{4}{|l|}{ Einträge } \\
\hline Atmosphärische Deposition ${ }^{\mathrm{a}} \mathrm{b}$ & $1-\mathbf{1}-20$ & $0,4-1,2$ & $0,1-\mathbf{0 , 2}-1,1$ \\
\hline Phosphat-Dünger ${ }^{\mathrm{c}}$ & $0,28-\mathbf{2 , 5}-5,6$ & $0,0-\mathbf{1 , 4}-\mathbf{3 , 7}$ & $\mathbf{0 , 7 4}$ \\
\hline Klärschlamm ${ }^{\mathrm{b}}$ & n.v. & & 0,05 \\
\hline Gülle ${ }^{b}$ & n.v. & n.v. & $\mathbf{0 , 0 1}$ \\
\hline Kalkung ${ }^{\mathrm{b}}$ & n.v. & n.v. & $\mathbf{0 , 0 7}$ \\
\hline \multicolumn{4}{|l|}{ Austräge } \\
\hline Ernte $^{\mathrm{bc}}$ & $\mathbf{0 , 3}$ & $0,1-1,2$ & 0,2 \\
\hline Versickerung ${ }^{\mathrm{b}} \mathrm{c}$ & n.v. & $0,15-0,9$ & 2,56 \\
\hline
\end{tabular}

${ }^{a}$ diese Studie

${ }^{\mathrm{b}}$ Kamermann et al. (2015)

${ }^{\mathrm{c}}$ Six und Smolders (2014)

oder für den Eintrag von Stoffen zur Veränderung des pHWertes sowie des Redoxmilieus, wie Sauerstoff oder Nitrat.

Für 16 BDF ließen sich Cd-Frachten aus der atmosphärischen Deposition berechnen. Die meisten Zeitreihen zeigten über den Zeitraum 1982 bis 2014 eine stetige Verringerung der Cd-Konzentrationen zwischen $1,0 \mathrm{~g} /$ (ha $\cdot$ a) und maximal $20 \mathrm{~g} /($ ha $\cdot$ a) auf aktuelle Werte zwischen $0,1 \mathrm{~g} /(\mathrm{ha} \cdot \mathrm{a})$ und maximal $1,1 \mathrm{~g} /(\mathrm{ha} \cdot \mathrm{a})$. Diese Werte liegen im Bereich bundesweiter Abschätzungen von Ilyin et al. (2016), die Depositionswerte für NW-Deutschland zwischen $0,3 \mathrm{~g} \mathrm{Cd} / \mathrm{ha}$ und $0,6 \mathrm{~g} \mathrm{Cd} / \mathrm{ha}$ pro Jahr angeben. Auch Six und Smolders (2014) haben für Deutschland einen mittleren Cd-Eintrag durch Deposition von $0,25 \mathrm{~g} /(\mathrm{ha} \cdot \mathrm{a})$ bestimmt. In Tab. 3 sind die berechneten und der Literatur entnommenen CdFrachten für Böden in Nordwestdeutschland zusammengefasst. Neben der Verringerung der Cd-Einträge aus der Atmosphäre lassen sich auch kleiner werdende Einträge aus Phosphat-Düngern beobachten.

Für jede BDF ergeben sich eigene Sickerwasserraten, sodass zwischen $3 \%$ und $45 \%$ der Cd-Frachten aus der Deposition in das Grundwasser gelangen können. Somit können bei einer aktuellen Deposition mit Werten von $0,1 \mathrm{Cd} /(\mathrm{ha} \cdot \mathrm{a})$ bis $1,1 \mathrm{Cd} /(\mathrm{ha} \cdot \mathrm{a}) \mathrm{Cd}-F r a c h t e n$ zwischen $0,01 \mathrm{~g} \mathrm{Cd} /(\mathrm{ha} \cdot \mathrm{a})$ und $0,4 \mathrm{~g} \mathrm{Cd} /(\mathrm{ha} \cdot \mathrm{a})$ potenziell das Grundwasser erreichen. Prozesse wie Ausfällung und Sorption von $\mathrm{Cd}$ sind dabei nicht berücksichtigt, würden aber die Einträge in das Grundwasser verringern. Dass solche konzentrationsmindernden Prozesse eine Rolle spielen, zeigen die tatsächlich gemessenen Cd-Konzentrationen im Sickerwasser der Bodendauerbeobachtungsflächen, denn sie waren meist gering oder zeigten eine Verringerung mit der Zeit. Die jeweils zuletzt gemessenen Werte für $\mathrm{Cd}$ liegen bei den BDF-F zwischen $0,12 \mu \mathrm{g} / \mathrm{l}$ und $1,1 \mu \mathrm{g} / \mathrm{l}$. Die Werte liegen unterhalb des 90. Perzentils der Cd-Konzentrationen im oberflächennahen Grundwasser unter Wald von 1,8 $\mu \mathrm{g} / \mathrm{l}$ im Fuhrberger Feld (Engel 2002). Ebenso sind sie im Rahmen des Hintergrundwertes im Sickerwasser unter Forst im Norddeutschen Tiefland als 90. Perzentil von 2,7 $\mu \mathrm{g} / \mathrm{l}$ (Duijnisveld et al. 2008). Bei den BDF-L sind die zuletzt gemessenen Cd-Konzentrationen des Sickerwassers $<0,35 \mu \mathrm{g} / \mathrm{l}$, was ebenfalls im Bereich der Hintergrundkonzentrationen für Ackerstandorte von $2,4 \mu \mathrm{g} / \mathrm{l}$ nach Engel (2002) bzw. 0,76 $\mu \mathrm{g} / \mathrm{l}$ nach Duijnisveld et al. (2008) liegt. Generell gelangen an Waldstandorten größere Frachten aus der Atmosphäre in den Untergrund als auf Freiland, da die Bäume durch die Blattoberfläche mehr Stoffe aufnehmen, was den bereits genannten Auskämmeffekt darstellt (Keuffel-Türk et al. 2012).

Daraus lässt sich zusammenfassen, dass die aktuellen Cd-Frachten aus der Deposition keinen wesentlichen Einfluss auf die Cd-Konzentrationen im Grundwasser haben. Eine erhöhte Deposition in der Vergangenheit kann jedoch zu einer Anreicherung des Cd im Boden geführt haben, das, abhängig vom hydrochemischen Milieu des Sickerwassers, mobilisiert werden kann.

Die Auswertung der Grundwasseranalysen weist darauf hin, dass die Cd-Konzentrationen im Zusammenhang mit der Landnutzung sowie der mineralischen Zusammensetzung des Grundwasserleiters steht. Niedersachsen weist Regionen auf, die landwirtschaftlich intensiv genutzt werden und in denen es zu einem gesteigerten Einsatz an Stickstoff- und Phosphat-Düngern kommt (Wendland et al. 1993). Stickstoffüberschüsse und Versickerung führen zu erhöhten Nitratgehalten im Sickerwasser, die schließlich das Grundwasser erreichen (Köhler et al. 2006; Wendland et al. 1993). Aus diesem Grund hatte das Land Niedersachsen in der Maßnahmenkulisse „Nitratreduktion“ (MU 2015b) Gebiete festgelegt (Abb. 6), um dort Maßnahmen zur Verbesserung herbeizuführen. Aus der Karte wird ersichtlich, dass die Messstellen mit $\mathrm{Cd}>0,5 \mu \mathrm{g} / \mathrm{l}$ zum Großteil in diesen Gebieten liegen. Damit lässt sich der Rückschluss ziehen, dass durch die Kombination aus Ni- 
trateintrag über Dünger, geogenem Cd in Sedimenten des Grundwasserleiters und die fortschreitende Pyritoxidation im Grundwasser Niedersachsens erhöhte Cd-Konzentrationen im Grundwasser in diesen landwirtschaftlich geprägten Gebieten verursachen. Allerdings wurden keine weiteren Schwermetalle in auffälligen Konzentrationen festgestellt, wie sie in anderen Studien in Deutschland beobachtet wurden (z. B. Cremer 2002; Riedel und Kübeck 2018; van Berk und $\mathrm{Fu} 2017)$.

Die negative Korrelation des pH-Werts mit Cd-Konzentrationen im Grundwasser kann zum einen die Freisetzung von Cd aus dem Pyrit oder seine Desorption vom Pyrit während der autotrophen Denitrifikation widerspiegeln, gleichwohl ist eine Desorption des Cd von anderen Mineralphasen auch allein aufgrund sinkender pH-Werte möglich (Kubier et al. 2019). Solche Gebiete niedriger $\mathrm{pH}$-Werte sieht man unter forstwirtschaftlicher Nutzung (Schilli et al. 2011).

\section{Schlussfolgerungen und Zusammenfassung}

Die möglichen anthropogenen, flächendeckend auftretenden Cd-Einträge im Untersuchungsgebiet (siehe Abb. 1) umfassen Phosphat-Dünger, Klärschlamm und Deposition von Industrieemissionen (Knappe et al. 2008). Es liegen keine ausreichend detaillierten Kenntnisse zu Art und Menge der ausgebrachten Phosphat-Dünger vor, um deren Bedeutung für Cd-Einträge abschätzen zu können. Dadurch können Grundwasseranalysen mit erhöhten Cd-Konzentrationen, die als anthropogen beeinflusst eingeschätzt werden, nicht eindeutig dem Szenario II „Geogener Ursprung, anthropogen induzierte Mobilisierung" oder dem Szenario III „Anthropogener Eintrag“ (Tab. 1) zugeordnet werden. Informationen zu Phosphatsalden, die auf Landkreisebene basieren, sind LWK Niedersachsen (2016) und Wiesler et al. (2015) zu entnehmen. Demnach ist nicht auszuschließen, dass Cd aus der Landwirtschaft ins Grundwasser eingetragen wird. Allerdings zeigt die Auswertung der BDF, dass $\mathrm{Cd}$ im Sickerwasser unter ungedüngtem Forst deutlich erhöht sein kann und somit außer dem Eintrag durch Dünger weitere Quellen anzunehmen sind.
Es wurden bei der Auswertung der Bodenanalysen der BDF keine Hinweise auf einen bedeutenden direkten $\mathrm{Cd}$ Eintrag festgestellt; die Cd-Frachten aus der atmosphärischen Deposition haben sich mit der Zeit verringert (Keuffel-Türk et al. 2012). Stattdessen zeigen die Grundwasseranalysen zusammen mit der Auswertung der Analysen von Boden-Dauerbeobachtungsflächen, dass die Cd-Konzentration im Grundwasser durch verschiedene Faktoren gesteuert wird. Insgesamt können Kriterien abgeleitet werden, welche zu erhöhten Cd-Konzentrationen beitragen (Tab. 4). Darunter fallen landwirtschaftlich genutzte Gebiete, organisch geprägte Gebiete wie Wald, Moore und Marschen sowie allgemein Bereiche der Grundwasserneubildung.

Cadmiumkonzentrationen im Grundwasser Niedersachsens und Bremens wurden an 6275 Grundwasser-Messstellen gemessen und liegen zwischen $<0,002 \mu \mathrm{g} / \mathrm{l}$ und 5,9 $\mu \mathrm{g} / \mathrm{l}$. Im Mittel betragen Cd-Hintergrundwerte im Grundwasser der Niederungen und Marschen $0,13 \mu \mathrm{g} / \mathrm{l}$ und in den Geesten $0,36 \mu \mathrm{g} / \mathrm{l}$. Der Schwellenwert für Cd gemäß Grundwasserverordnung beträgt $0,5 \mu \mathrm{g} / \mathrm{l}(\mathrm{GrwV} 2017)$. Dadurch wurde aufgrund der Schwellwertüberschreitung einiger Messstellen im Zuge der Bewertung nach Wasserrahmenrichtlinie der chemische Zustand mehrerer Grundwasserkörper als schlecht eingestuft. Die Ergebnisse des in der Folge initiierten Forschungsprojektes zeigen, dass aktuell kein nennenswerter Cd-Eintrag durch atmosphärische Deposition erfolgt. Durch Luftreinhaltemaßnahmen nimmt die atmosphärische Deposition seit Jahren ab. Darüber hinaus konnte nicht aufgedeckt werden, ob Phosphat-Dünger, wie in der Vergangenheit gemessen (Kamermann et al. 2015; Six und Smolders 2014), aktuell weiterhin zu bedeutenden direkten CdEinträgen führen. Neben den Einträgen wurde festgestellt, dass andere Parameter eher dazu führen, dass mineralisch gebundenes $\mathrm{Cd}$ mobilisiert werden kann, etwa im Gebiet der Geesten unter landwirtschaftlicher Nutzung. Aus diesem Grund ist zu klären 1. ob es notwendig ist, Maßnahmen zur Verbesserung der Situation zu ergreifen und 2. welche Maßnahmen dafür geeignet sind.

$\mathrm{Zu}$ 1. ist anzumerken, dass der in Deutschland ausschlaggebende Schwellenwert der Grundwasserverordnung mit $0,5 \mu \mathrm{g} / \mathrm{l}$ unterhalb des Grenzwertes der Trinkwasserordnung

Tab. 4 Beispiele für Kriterien erhöhter Cadmium-Konzentrationen im Grundwasser

Table 4 Examples of criteria for elevated cadmium concentrations in groundwater

\begin{tabular}{|c|c|c|}
\hline Kriterium & Merkmale & Für Cd relevante Prozesse \\
\hline $\begin{array}{l}\text { Nutzung Land- } \\
\text { wirtschaft }\end{array}$ & $\begin{array}{l}\text { Geringer } \mathrm{pH} \text {, hohe Konzentrationen an Nitrat, } \\
\text { Sauerstoff und weiteren Schwermetallen }\end{array}$ & $\begin{array}{l}\text { Freisetzung durch Pyrit-Oxidation; durch Säureeintrag Mobilisierung } \\
\text { z. B. aus Eisenoxiden; ggf. direkter Eintrag aus Düngung (P-Dünger) }\end{array}$ \\
\hline Nutzung Wald & $\begin{array}{l}\text { Geringer } \mathrm{pH} \text {, hohe Konzentrationen an DOC und } \\
\text { weiteren Schwermetallen }\end{array}$ & $\begin{array}{l}\text { Mobilisierung durch Säureeintrag, ggf. direkter Eintrag aus Depositi- } \\
\text { on (Auskämmeffekt) }\end{array}$ \\
\hline $\begin{array}{l}\text { Standort Moor/ } \\
\text { Marsch }\end{array}$ & $\begin{array}{l}\text { Reduzierendes Milieu: Fehlen von } \mathrm{NO}_{3}, \mathrm{O}_{2} \text {, teils } \\
\mathrm{SO}_{4} \text {, dafür Fe, teils DOC, } \mathrm{HCO}_{3} \text { oder } \mathrm{CH}_{4} \text { erhöht }\end{array}$ & $\begin{array}{l}\text { Mobilisierung durch Säureeintrag, Freisetzung aus Sulfiden und orga- } \\
\text { nischem Material, Transport als stabile an-/organische Komplexe }\end{array}$ \\
\hline $\begin{array}{l}\text { Grundwasser- } \\
\text { Neubildung }\end{array}$ & $\begin{array}{l}\text { Saisonale Schwankungen von } \mathrm{pH}, \mathrm{Cd}, \mathrm{Ni}, \mathrm{Zn} \text {, } \\
\mathrm{SO}_{4} / \mathrm{Cl}, \mathrm{O}_{2}\end{array}$ & $\begin{array}{l}\text { Wechselnde De-/Sorption durch Schwankungen im Grenz-pH-Bereich } \\
\text { und Redoxmilieu, auch bei Ni und Zn }\end{array}$ \\
\hline
\end{tabular}


von 3,0 $\mu \mathrm{g} / \mathrm{l}$ (TrinkwV 2018) und der Schwellenwerte der meisten anderen EU-Mitgliedsstaaten liegt. Dies entspringt der Systematik, dass für die Grundwasserverordnung der empfindlichste Rezeptor als Maß genommen worden ist. Da Grundwasser Seen und Flüsse über den unterirdischen Abfluss speist, hat dies aus der Perspektive des vorbeugenden und flächendeckenden Grundwasserschutzes seine Berechtigung. Der Schwellenwert stellt auch keinen Grenzwert wie die Werte aus dem Lebensmittelbereich dar. Cadmiumkonzentrationen unterhalb des Schwellenwertes gelten als unbedenklich, während bei Überschreitungen zuerst der Besorgnisgrundsatz zu prüfen ist und ggf. Maßnahmen ergriffen werden müssen.

Für 2. ist festzustellen, dass die erhöhten Cd-Konzentrationen überwiegend im Bereich der landwirtschaftlichen genutzten Flächen auftreten, besonders in Geesten mit Nitratüberschüssen. Die an die Denitrifikation gekoppelte $\mathrm{Cd}-$ Freisetzung ins Grundwasser, etwa aus Pyrit, entsteht unter bestimmten hydrochemischen Rahmenbedingungen. Somit stellen die Cd-Konzentrationen im Grundwasser ein Symptom der Landwirtschaft dar, was durch die Kongruenz der Flächen mit erhöhtem $\mathrm{Cd}$ und der Nitratkulisse deutlich wird, sodass als Maßnahmenfelder vorgeschlagen werden können:

- Überarbeitung der Nitratkulisse: Hier ist zu prüfen, ob es Gebiete gibt, in denen Cd im Grundwasser erhöht ist, aber keine Nitrat-Maßnahmenkulisse vorliegt.

- Ggf. Anheben der pH-Werte im Bereich der Ackerböden.

- Neubewertung des chemischen Zustands der Grundwasserkörper. Neben dem Schwellenwert sollten die Hintergrundwerte, die für die hydrogeologisch berechneten Teilräume ermittelt wurden, berücksichtigt werden.

- Beratung der Landwirtschaft bei der Anwendung von Phosphat-Düngern zur Minimierung möglicher Cd-Einträge auf diesem Wege.

Die Bewertung des Umweltbundesamtes zu den Nitrateinträgen (Bach et al. 2016) zeigt den gegenwärtigen Zustand und weitere denkbare Maßnahmen.

Die statistischen Auswertungen der Grundwasserdaten bestätigen die in dieser Untersuchung entwickelten Szenarien. Sie zeigen, dass Cd durch Änderungen des pH-Wertes und des Redoxpotenzials ins Sicker- bzw. Grundwasser freigesetzt wird und dass dies unter landwirtschaftlicher und forstwirtschaftlicher Nutzung stattfindet. Da Cd-Einträge durch Deposition zurückgehen, Cd-Einträge aus PhosphatDüngern seit den 1980ern ebenfalls zurückgehen, aktuell jedoch weder nachweisbar noch zu vernachlässigen sind, sind erhöhte Cd-Konzentrationen im Grundwasser überwiegend eine Folge der Nutzung, also ein Sekundäreffekt.

Die Bedeutung dieser Studie liegt darin, dass grundlegend bekannte Prozesse und Zusammenhänge flächenhaft für Nordwestdeutschland betrachtet werden. Statisti- sche Betrachtungen und die Verschneidung mit Informationen zur Nutzung und Geologie/Hydrogeologie erlauben die Ableitung von plausiblen Maßnahmengebieten. Dass diese innerhalb der bekannten Maßnahmenkulisse zur Nitratreduktion liegt (Abb. 6), bestätigt die Nitratkulisse zum einen, zeigt aber auch, dass es innerhalb dieser noch Schwerpunktgebiete gibt, in denen man Maßnahmen zur Erreichung von Umweltzielen konzentrieren könnte. 


\section{Anhang}

Abb. 7 Cadmium-Hintergrundwerte der Hydrogeologischen

Teilräume. Die farbliche Zuordnung entspricht in steigender Reihenfolge der Cd-Werte von $0,01 \mu \mathrm{g} / \mathrm{l}$ (grün), über $0,2 \mu \mathrm{g} / \mathrm{l}$ und $0,3 \mu \mathrm{g} / 1$ (gelb) bis $0,98 \mu \mathrm{g} / \mathrm{l}$ (rot)

Fig. 7 Cadmium background levels of the hydrogeological subareas. The color-coded attribution corresponds to the increasing order of $\mathrm{Cd}$ values from $0.01 \mu \mathrm{g} / 1$ (green), to $0.2 \mu \mathrm{g} / 1$ to $0,3 \mu \mathrm{g} / 1$ (yellow) to $0,98 \mu \mathrm{g} / 1$ (red)

\begin{tabular}{|c|c|c|c|c|}
\hline $\begin{array}{l}\text { Teilraum- } \\
\text { Nummer }\end{array}$ & Teilraum-Name & $\begin{array}{l}\text { Anzahl } \\
\text { Messstellen }\end{array}$ & $\begin{array}{l}\text { Anzahl Messstellen } \\
\text { Cd }>0,5 \mu \mathrm{g} / \mathrm{l}\end{array}$ & $\begin{array}{l}\text { Hintergrundwert } \\
\mathrm{Cd}[\mu \mathrm{g} / \mathrm{l}]\end{array}$ \\
\hline 01102 & Ostfriesische Inseln & 87 & 3 & 0,23 \\
\hline 01103 & Ostfriesische Watten & 0 & 0 & $X$ \\
\hline 01204 & Elbmarsch & 55 & 15 & 0,01 \\
\hline 01205 & Unterweser Marsch & 119 & 8 & 0,01 \\
\hline 01206 & Ostfriesische Marsch & 115 & 6 & 0,01 \\
\hline 01301 & Elbe Niederung & 140 & 15 & 0,23 \\
\hline 01304 & Mittelweser-Aller-Leine Niederung & 732 & 60 & 0,13 \\
\hline 01305 & Ems-Vechte-Niederung & 316 & 42 & 0,18 \\
\hline 01306 & Bourtanger Moorniederung & 63 & 4 & 0,10 \\
\hline 01307 & Hunte-Leda-Moorniederung & 143 & 6 & 0,03 \\
\hline 01308 & Quakenbrücker Becken & 119 & 14 & 0,27 \\
\hline 01309 & $\begin{array}{l}\text { Diepholzer Moorniederung und } \\
\text { Rinne von Hille }\end{array}$ & 106 & 12 & 0,13 \\
\hline 01310 & Hamme Moornierung & 25 & 0 & 0,03 \\
\hline 01313 & Wümme Niederung & 64 & 7 & 0,27 \\
\hline 01314 & Drömling und Ohre Niederung & 24 & 1 & 0,13 \\
\hline 01501 & Oldenburgisch-Ostfriesische Geest & 376 & 15 & 0,03 \\
\hline 01502 & Sögeler Geest & 126 & 41 & 0,35 \\
\hline 01503 & Cloppenburger Geest & 123 & 29 & 0,48 \\
\hline 01504 & Syker Geest & 193 & 61 & 0,78 \\
\hline 01505 & Itterbecker Geest & 24 & 10 & 0,36 \\
\hline 01506 & Lohner Geest & 1 & 0 & 0,36 \\
\hline 01507 & Emsbürener Geest & 9 & 1 & 0,98 \\
\hline 01508 & Lingener Höhe & 25 & 5 & 0,36 \\
\hline 01509 & Ankumer Höhe & 55 & 5 & 0,36 \\
\hline 01510 & Dammer Berge & 20 & 1 & 0,36 \\
\hline 01511 & Kellenberg Geest & 16 & 4 & 0,36 \\
\hline 01512 & Diepenauer Geest & 18 & 9 & 0,03 \\
\hline 01513 & Böhrde Geest & 30 & 3 & 0,36 \\
\hline 01514 & Nienburg-Neustädter Geest & 50 & 8 & 0,36 \\
\hline 01515 & Hannoversche Moorgeest & 80 & 5 & 0,03 \\
\hline 01516 & Wedemark Geest & 16 & 0 & 0,36 \\
\hline 01517 & Isernhagener Rücken & 1 & 0 & 0,36 \\
\hline 01518 & Burgdorfer Geest & 109 & 24 & 0,98 \\
\hline 01519 & Papenteich Geest & 15 & 2 & 0,06 \\
\hline 01520 & Bederkesa Geest & 267 & 43 & 0,54 \\
\hline 01521 & Zevener Geest & 89 & 9 & 0,12 \\
\hline 01522 & Lüneburger Heide West & 573 & 45 & 0,18 \\
\hline 01523 & Lüneburger Heide Ost & 434 & 70 & 0,31 \\
\hline 01524 & $\begin{array}{l}\text { Altmark mit Cölbitz-Letzlinger } \\
\text { Heide }\end{array}$ & 6 & 1 & 0,31 \\
\hline 01525 & Langendorfer Geest & 1 & 0 & 0,31 \\
\hline
\end{tabular}


Abb. 7 (Forsetzung) CadmiumHintergrundwerte der Hydrogeologischen Teilräume. Die farbliche Zuordnung entspricht in steigender Reihenfolge der Cd-Werte von $0,01 \mu \mathrm{g} / \mathrm{l}$ ( grün), über $0,2 \mu \mathrm{g} / \mathrm{l}$ und $0,3 \mu \mathrm{g} / \mathrm{l}(\mathrm{gelb})$ bis $0,98 \mu \mathrm{g} / \mathrm{l}($ rot $)$

Fig. 7 (continuation) Cadmium background levels of the hydrogeological subareas. The colorcoded attribution corresponds to the increasing order of $\mathrm{Cd}$ values from $0.01 \mu \mathrm{g} / \mathrm{l}$ (green), to $0.2 \mu \mathrm{g} / 1$ to $0,3 \mu \mathrm{g} / 1$ (yellow) to $0,98 \mu \mathrm{g} / \mathrm{l}(\mathrm{red})$

\begin{tabular}{|c|c|c|c|c|}
\hline $\begin{array}{l}\text { Teilraum- } \\
\text { Nummer }\end{array}$ & Teilraum-Name & $\begin{array}{l}\text { Anzahl } \\
\text { Messstellen }\end{array}$ & $\begin{array}{l}\text { Anzahl Messstellen } \\
\text { Cd }>0,5 \mu \mathrm{g} / \mathrm{l}\end{array}$ & $\begin{array}{l}\text { Hintergrundwert } \\
\text { Cd }[\mu \mathrm{g} / \mathrm{l}]\end{array}$ \\
\hline 01526 & Höhbeck & 7 & 0 & 0,31 \\
\hline 02101 & $\begin{array}{l}\text { Niederung der Ems und der oberen } \\
\text { Lippe }\end{array}$ & 23 & 1 & 0,42 \\
\hline 02207 & Ochtruper Sattel & 0 & 0 & $\mathrm{X}$ \\
\hline 02208 & Osning und Thieberg & 14 & 1 & 0,07 \\
\hline 05101 & Bentheimer Berge & 8 & 3 & 0,26 \\
\hline 05102 & Ibbenbühren-Osnabrücker Bergland & 89 & 1 & 0,26 \\
\hline 05103 & Wiehengebirge & 31 & 1 & 0,54 \\
\hline 05104 & $\begin{array}{l}\text { Südliches Vorland des } \\
\text { Wiehengebirges }\end{array}$ & 7 & 2 & 0,54 \\
\hline 05105 & Herforder Mulde & 3 & 0 & 0,07 \\
\hline 05106 & Stemweder Berg & 3 & 0 & 0,42 \\
\hline 05107 & $\begin{array}{l}\text { Kreidemergel des nördlichen } \\
\text { Wiehengebirgsvorlandes }\end{array}$ & 0 & 0 & $\mathrm{X}$ \\
\hline 05109 & Herford-Hamelner Bergland & 40 & 2 & 0,26 \\
\hline 05110 & Steinheim-Ottensteiner Hochfläche & 33 & 0 & 0,18 \\
\hline 05112 & Bückebergvorland & 13 & 6 & 0,42 \\
\hline 05113 & Calenberger Bergland & 63 & 5 & 0,54 \\
\hline 05114 & Calenberger Lössbörde & 89 & 8 & 0,42 \\
\hline 05115 & Hilsmulde & 60 & 5 & 0,18 \\
\hline 05116 & Leinetalgraben & 103 & 5 & 0,18 \\
\hline 05117 & $\begin{array}{l}\text { Innerste Bergland und nördliches } \\
\text { Harzvorland }\end{array}$ & 164 & 24 & 0,18 \\
\hline 05118 & Sackmulde & 20 & 3 & 0,54 \\
\hline 05119 & $\begin{array}{l}\text { Braunschweig-Hildesheimer } \\
\text { Lössbörde }\end{array}$ & 34 & 11 & 0,24 \\
\hline 05120 & Wolfenbütteler Hügelland & 15 & 0 & 0,07 \\
\hline 05121 & Hase-Else-Werre Talaue & 25 & 0 & 0,06 \\
\hline 05122 & Oberweser Talaue & 41 & 0 & 0,06 \\
\hline 05123 & Leine-Innnerste Talaue & 213 & 3 & 0,42 \\
\hline 05124 & Oker Talaue & 32 & 3 & 0,06 \\
\hline 05125 & Homburger Zechsteingebiet & 0 & 0 & $\mathrm{X}$ \\
\hline 05127 & Karbon im Osnabrücker Bergland & 0 & 0 & $\mathrm{X}$ \\
\hline 05128 & Karbon im Teutoburger Wald & 0 & 0 & $\mathrm{X}$ \\
\hline 05129 & Zechstein im Teutoburger Wald & 2 & 0 & 0,41 \\
\hline 05201 & Fulda-Werra-Bergland und Solling & 121 & 7 & 0,12 \\
\hline 05301 & Subherzyne Mulde & 9 & 1 & 0,09 \\
\hline 05302 & Oschersleben-Bernburger Scholle & 16 & 4 & 0,07 \\
\hline 05303 & Schönebeck-Weferlinger Triasplatte & 10 & 4 & 0,09 \\
\hline 05304 & $\begin{array}{l}\text { Wolfsburger Hügelland und } \\
\text { Lappwald }\end{array}$ & 35 & 10 & 0,09 \\
\hline 05305 & Elm & 15 & 1 & 0,07 \\
\hline 05401 & $\begin{array}{l}\text { Zechsteinrand der Thüringischen } \\
\text { Senke }\end{array}$ & 19 & 2 & 0,41 \\
\hline 05402 & $\begin{array}{l}\text { Buntsandsteinumrandung der } \\
\text { Thüringischen Senke }\end{array}$ & 82 & 9 & 0,14 \\
\hline 08301 & Harz & 72 & 17 & $(0,73)$ \\
\hline 08302 & Magdeburg-Flechtinger Hochlage & 0 & 0 & $\mathrm{X}$ \\
\hline
\end{tabular}


Danksagung Das Forschungsprojekt wurde aus Zuwendungen des Landes Niedersachsen zum Maßnahmenprogramm im Bereich Grundwasser zur Umsetzung der EU-Wasserrahmenrichtlinie finanziert. Wir danken dem Niedersächsischen Landesbetrieb für Wasserwirtschaft, Küsten- und Naturschutz (NLWKN), dem Landesamt für Bergbau, Energie und Geologie (LBEG), dem Senator für Umwelt, Bau und Verkehr (SUBV) Bremen, der Stadt Hannover und der Nordwestdeutschen Forstlichen Versuchsanstalt (NW-FVA) für die Bereitstellung der chemischen Daten.

Funding Open Access funding enabled and organized by Projekt DEAL.

Open Access Dieser Artikel wird unter der Creative Commons Namensnennung 4.0 International Lizenz veröffentlicht, welche die Nutzung, Vervielfältigung, Bearbeitung, Verbreitung und Wiedergabe in jeglichem Medium und Format erlaubt, sofern Sie den/die ursprünglichen Autor(en) und die Quelle ordnungsgemäß nennen, einen Link zur Creative Commons Lizenz beifügen und angeben, ob Änderungen vorgenommen wurden.

Die in diesem Artikel enthaltenen Bilder und sonstiges Drittmaterial unterliegen ebenfalls der genannten Creative Commons Lizenz, sofern sich aus der Abbildungslegende nichts anderes ergibt. Sofern das betreffende Material nicht unter der genannten Creative Commons Lizenz steht und die betreffende Handlung nicht nach gesetzlichen Vorschriften erlaubt ist, ist für die oben aufgeführten Weiterverwendungen des Materials die Einwilligung des jeweiligen Rechteinhabers einzuholen.

Weitere Details zur Lizenz entnehmen Sie bitte der Lizenzinformation auf http://creativecommons.org/licenses/by/4.0/deed.de.

\section{Literatur}

Ad-hoc-Arbeitsgruppe Hydrogeologie: Regionale Hydrogeologie von Deutschland. Geologisches Jahrbuch, Reihe A, Bd. A 163. Schweizerbart, Stuttgart, S. 45 (2016)

Aziz, J., Ling, M., Rifai, H.S., Newell, C.J., Gonzales, J.R.: MAROS: a decision support system for optimizing 464 monitoring plans. Ground Water 41, 355-367 (2004)

Bach, M., Klement, L., Häußermann, U.: Bewertung von Maßnahmen zur Verminderung von Nitrateinträgen in die Gewässer auf Basis regionalisierter Stickstoff-Überschüsse. Teil I: Beitrag zur Entwicklung einer Ressortübergreifenden Stickstoffstrategie. Umweltbundesamt, Dessau-Roßlau, S. 51 (2016)

Bergmann, A., Van Straaten, L., Van Berk, W., Dietrich, P., Franko, U., Kiefer, J.: Konsequenzen nachlassenden Nitratabbauvermögens in Grundwasserleitern. Deutscher Verein des Gas- und Wasserfaches e.V. (DVGW), Bonn, S. 179 (2013). Abschlussbericht

van Berk, W., Fu, Y.J.: Redox roll-front mobilization of geogenic uranium by nitrate input into aquifers: risks for groundwater resources. Environ. Sci. Technol. 51, 337-345 (2017)

Böhlke, J.K.: Groundwater recharge and agricultural contamination. Hydrogeol J 10, 153-179 (2002)

Budziak, D., Kubier, A., Elbracht, J.: Spurenelemente im Grundwasser Niedersachsens. In: Wolff, J. (Hrsg.) Grundwasserschutz im Spannungsfeld zwischen Nachhaltigkeit und Ökonomie. Tagungsband - Niedersächsisches Grundwasserkolloquium, 15./16. Februar 2017 in Braunschweig. Teil I, Jg. 2017 Heft 1 Zentralblatt für Geologie und Paläontologie. S. 232. Schweizerbart'sche Verlagsbuchhandlung, Stuttgart (2017in)

Carrillo-Gonzalez, R., Simunek, J., Sauve, S., Adriano, D.: Mechanisms and pathways of trace element mobility in soils. Adv. Agron. 91, 111-178 (2006)

Cohen, J.: Statistical power analysis for the behavioral sciences, 2. Aufl. Erlbaum, Mahwah (1988)
Cremer, N.: Schwermetalle im Grundwasser Nordrhein-Westfalens unter besonderer Berücksichtigung des Nickels in tieferen Grundwasserleitern der Niederrheinischen Bucht. Besondere Mitteilungen zum Deutschen Gewässerkundlichen Jahrbuch, Bd. 60. Landesumweltamt Nordheim-Westfalen, Essen, S. 178 (2002)

Duijnisveld, W.H.M., Godbersen, L., Dilling, J., Gäbler, H.-E., Utermann, J., Klump, G., Scheeder, G.: Ermittlung flächenrepräsentativer Hintergrundkonzentrationen prioritärer Schadstoffe im Bodensickerwasser. Forschungsbericht des Umweltbundesamtes. Bundesanstalt für Geowissenschaften und Rohstoffe, Hannover, S. 163 (2008)

Elbracht, J., Meyer, R., Reutter, E.: Hydrogeologische Räume und Teilräume in Niedersachsen, 2. Aufl. GeoBerichte, Bd. 3. Landesamt für Bergbau, Energie und Geologie, Hannover, S. 107 (2016)

Engel, H.J.: Hintergrundkonzentrationen von Spurenelementen und Schwermetallen im oberflächennahen Grundwasser: Literaturüberblick und Feldstudie (2002). Univ. Hannover

ESRI: ArcGIS desktop 10. Environmental Systems Research Institute, Redlands (2018)

GrwV: Grundwasserverordnung vom 9. November 2010 (BGBl. I S. 1513), die zuletzt durch Artikel 1 der Verordnung vom 4. Mai 2017 (BGBl. I S. 1044) geändert worden ist. 3 S.; Bundesrepublik Deutschland, Bonn (2017)

Herms, U., Brümmer, G.: Einflußgrößen der Schwermetallöslichkeit und -bindung in Böden. Z Pflanzernaehr Bodenk 147, 400-424 (1984)

Hinsby, K., de Melo, M.T.C., Dahl, M.: European case studies supporting the derivation of natural background levels and groundwater threshold values for the protection of dependent ecosystems and human health. Sci. Total Environ. 401, 1-20 (2008)

Höper, H., Meesenburg, H.: Tagungsband 20 Jahre Bodendauerbeobachtung in Niedersachsen. GeoBerichte, Bd. 23. Landesamt für Bergbau, Energie und Geologie, Hannover, S. 254 (2012)

Houben, G.J., Sitnikova, M.A., Post, V.E.A.: Terrestrial sedimentary pyrites as a potential source of trace metal release to groundwater-A case study from the Emsland, Germany. Appl. Geochem. 76, 99-111 (2017)

Huschek, G., Krengel, D., Kayser, M., Bauriegel, A., Burger, H.: Länderübergreifende Auswertung von Daten der Boden-Dauerbeobachtung der Länder. Umweltbundesamt, Berlin, S. 104 (2004)

Ilyin, I., Gusev, A., Rozovskaya, O., Strijkina, I.: Transboundary Pollution by Heavy Metals and Persistent Organic Pollutants in 2014-Germany. EMEP/MSC-E Data Note, Bd. 5/2016., S. 33 (2016). European Monitoring and Evaluation Programme

Jorgensen, C.J., Jacobsen, O.S., Elberling, B., Aamand, J.: Microbial oxidation of pyrite coupled to nitrate reduction in anoxic groundwater sediment. Environ. Sci. Technol. 43, 4851-4857 (2009)

Kamermann, D., Groh, H., Höper, H.: Schwermetallein- und -austräge niedersächsischer Boden-Dauerbeobachtungsflächen Bd. 30 . Landesamt für Bergbau, Energie und Geologie, Hannover, S. 56 (2015)

Kendall, M.G.: Rank Correlation Methods. Charles Griffin, London (1975)

Keuffel-Türk, A., Jankowski, A., Scheler, B., Rademacher, P., Meesenburg, H.: Stoffeinträge durch Deposition. In: Höper, H., Meesenburg, H. (Hrsg.) Tagungsband 20 Jahre Bodendauerbeobachtung in Niedersachsen, Bd. 23, S. 19. Landesamt für Bergbau, Energie und Geologie, Hannover (2012)

Knappe, F., Mohler, S., Ostermayer, A., Lazar, S., Kaufmann, C.: Vergleichende Auswertung von Stoffeinträgen in Böden über verschiedene Eintragspfade. Forschungsbericht des Umweltbundesamtes, Dessau-Roßlau, S. 382 (2008)

Köhler, K., Duijnisveld, W.H.M., Böttcher, J.: Nitrogen fertilization and nitrate leaching into groundwater on arable sandy soils. J. Plant Nutr. Soil Sci. 169, 185-195 (2006)

Koopmann, S., Fröllje, H., Hamer, K., Kubier, A., Pichler, T.: EisenMangan-Anomalien im Grundwasser - Analyse der beeinflussenden Prozesse. Grundwasser 25(2), 113-126 (2020) 
Krishnamurti, G.S.R., Naidu, R.: Solid-solution equilibria of cadmium in soils. Geoderma 113, 17-30 (2003)

Kubier, A., Pichler, T.: Cadmium in groundwater-A synopsis based on a large hydrogeochemical data set. Sci Tot Environ 689, 831-842 (2019)

Kubier, A., Hamer, K., Pichler, T.: Cadmium background levels in groundwater in an area dominated by agriculture. Integr Environ Assess Manag 16, 103-113 (2020)

Kubier, A., Pichler, T., Hamer, K.: Cadmium im Grundwasser Niedersachsens. Abschlussbericht an das Niedersächsische Ministerium für Umwelt, Energie, Bauen und Klimaschutz, S. 97 (2018). Univ. Bremen

Kubier, A., Wilkin, R.T., Pichler, T.: Cadmium in soils and groundwater: a review. Appl. Geochem. 108, 104388 (2019)

Kunkel, R., Voigt, H.-J., Wendland, F., Hannappel, S.: Die natürliche, ubiquitär überprägte Grundwasserbeschaffenheit in Deutschland. Bd. 47. Schriften des Forschungszentrums Jülich, Jülich, S. 204 (2004)

LBEG: Geologische Übersichtskarte von Niedersachsen $1: 500.000$ (GUEK500) NIBIS Kartenserver, Landesamt für Bergbau, Energie und Geologie (2000). https://nibis.lbeg.de/cardomap3/public/ ogc .ash $x$ ? NodeId $=64 \&$ Service $=$ WMS $\&$ Reque st=GetCapabilities\&, Zugegriffen: 10.07.2018

LBEG: Hydrogeologische Karte von Niedersachsen 1 : 50000 (HK50). NIBIS Kartenserver, Landesamt für Bergbau, Energie und Geologie (2008). https://nibis.lbeg.de/cardomap3/public/ogc.ashx? NodeId=200\&Service $=$ WMS $\&$ Request $=$ GetCapabilities $\&, \quad \mathrm{Zu}-$ gegriffen: 10.07.2018

LBEG: Hydrogeologische Übersichtskarte von Niedersachsen $1: 200$ 000 (HUEK200). NIBIS Kartenserver, Landesamt für Bergbau, Energie und Geologie (2015). https://nibis.lbeg.de/cardomap3/ public/ogc .ashx ?NodeId=55\&Service $=$ WMS $\&$ Request $=$ GetCapa bilities\&, Zugegriffen: 10.07.2018

LBEG: Hydrogeologische Übersichtskarte von Niedersachsen $1: 500$ 000 (HUEK500) NIBIS Kartenserver, Landesamt für Bergbau, Energie und Geologie (2004). https://nibis.lbeg.de/cardomap3/ public/ogc.ashx? NodeId=194\&Service=WMS\&Request=GetCapa bilities\&, Zugegriffen: 10.07.2018

LBM-DE2012: Digitales Landbedeckungsmodell für Deutschland (CORINE Land Cover 10 ha). Referenzjahr 2012, Stand der Dokumentation: 07.01.2016; Bundesamt für Kartographie und Geodäsie

Lepeltier, C.: A simplified statistical treatment of geochemical data by graphical representation. Econ. Geol. 64(5), 538-550 (1969)

LWK Niedersachsen: Nährstoffbericht in Bezug auf Wirtschaftsdünger für Niedersachsen 2014/2015. Landwirtschaftskammer Niedersachsen, Oldenburg, S. 207 (2016)

Mann, H.B.: Non parametric test against trend. Econometrica 13, 245-259 (1945)

Mollema, P.N., Stuyfzand, P.J., Juhasz-Holterman, M.H.A., Van Diepenbeek, P.M.J.A., Antonellini, M.: Metal accumulation in an artificially recharged gravel pit lake used for drinking water supply. J. Geochem. Explor. 150, 35-51 (2015)

MU: Niedersächsischer Beitrag zu den Bewirtschaftungsplänen 2015a bis 2021 der Flussgebiete Elbe, Weser, Ems und Rhein. Niedersächsisches Ministerium für Umwelt, Energie und Klimaschutz, Hannover, S. 318 (2015a)

MU: Niedersächsischer Beitrag zu den Maßnahmenprogrammen 2015b bis 2021 der Flussgebiete Elbe, Weser, Ems und Rhein. Niedersächsisches Ministerium für Umwelt, Energie und Klimaschutz, Hannover, S. 303 (2015b)

MU: WRRL Grundwasser - Chemischer Zustand gesamt Grundwasser. Umweltkartenserver des Niedersächsischen Ministeri- ums für Umwelt, Energie und Klimaschutz (2018). https://www. umweltkarten-niedersachsen.de/Umweltkarten/?topic=Wasserrah menrichtlinie\&lang=de\&bgLayer=TopographieGrau\&catalogNo des $=\&$ layers=Chemischer_Zustand_gesamt_Grundwasser, Zugegriffen: 5. Juli 2018

NLWKN: Gewässerüberwachungssystem Niedersachsen (GÜN) - Güte- und Standsmessnetz Grundwasser. Grundwasser, Bd. 18. Niedersächsischer Landesbetrieb für Wasserwirtschaft, Küsten- und Naturschutz, Norden, S. 46 (2014)

Riedel, T., Kübeck, C.: Uranium in groundwater-A synopsis based on a large hydrogeochemical data set. Water Res 129, 29-38 (2018)

RL 2000/60/EG: Richtlinie 2000/60/EG des Europäischen Parlaments und des Rates vom 23. Oktober 2000 zur Schaffung eines Ordnungsrahmens für Maßnahmen der Gemeinschaft im Bereich der Wasserpolitik (EG Wasserrahmenrichtlinie). Amtsblatt der Europäischen Gemeinschaften L 327/1. Zuletzt geändert durch Richtlinie 2014/101/EU der Kommission vom 30. Oktober 2014

RL 2006/118/EG: Richtlinie 2006//118/EG des Europäischen Parlaments und des Rates vom 27. Dezember 2006 zum Schutz des Grundwassers vor Verschmutzung und Verschlechterung. Amtsblatt der Europäischen Gemeinschaften L 372/19. Zuletzt geändert durch Richtlinie 2014/80/EU der Kommission vom 20. Juni 2014

Schilli, C., Rinklebe, J., Lischeid, G., Kaufmann-Boll, C., Lazar, S.: Auswertung der Veränderungen des Bodenzustands für BodenDauerbeobachtungsflächen (BDF) und Validierung räumlicher Trends unter Einbeziehung anderer Messnetze. Teil B: Datenauswertung und Weiterentwicklung des Monitorings. Umweltbundesamt, Dessau-Roßlau, S. 131 (2011)

Six, L., Smolders, E.: Future trends in soil cadmium concentration under current cadmium fluxes to European agricultural soils. Sci Tot Environ 485, 319-328 (2014)

Spearman, C.: The proof and measurement of association between two things. Am. J. Psychol. 15, 72-101 (1904)

TrinkwV: Trinkwasserverordnung in der Fassung der Bekanntmachung vom 10. März 2016 (BGB1. I S. 459), die zuletzt durch Artikel 1 der Verordnung vom 3. Januar 2018 (BGB1. I S. 99) geändert worden ist. 45 S.; Bundesrepublik Deutschland, Berlin (2018)

Wagner, B., Walter, T., Himmelsbach, T., Clos, P., Beer, A., Budziak, D., Dreher, T., Fritsche, H.G., Hubschmann, M., Marczinek, S., Peters, A., Poeser, H., Schuster, H., Steinel, A., Wagner, F., Wirsing, G.: Hydrogeochemische Hintergrundwerte der Grundwässer Deutschlands als Web Map Service. Grundwasser 16(3), 155-162 (2011)

Wendland, F., Albert, H., Bach, M., Schmidt, R.: Atlas zum Nitratstrom in der Bundesrepublik Deutschland. Springer, Berlin, Heidelberg, New York, S. 75 (1993)

Wiesler, F., Hund-Rinke, K., Gäth, S., George, E., Greef, J.M., Hölzle, L.E., Holz, F., Hülsbergen, K.-J., Pfeil, R., Severin, K., Frede, H.-G., Blum, B., Schenkel, H., Horst, W., Dittert, K., Ebertseder, T., Osterburg, B., Philipp, W., Pietsch, M.: Anwendung von organischen Düngern und organischen Reststoffen in der Landwirtschaft. Wissenschaftlicher Beirat für Düngungsfragen beim Bundesministerium für Ernährung und Landwirtschaft, Bundesanstalt für Landwirtschaft und Ernährung, Bonn, S. 34 (2015)

Wriedt, G., de Vries, D., Eden, T., Federolf, C.: Regionalisierte Darstellung der Nitratbelastung im Grundwasser Niedersachsens. Grundwasser 24(1), 1-15 (2019)

Hinweis des Verlags Der Verlag bleibt in Hinblick auf geografische Zuordnungen und Gebietsbezeichnungen in veröffentlichten Karten und Institutsadressen neutral. 